\title{
A stochastic process modelling of maize phyllochron enables to characterize environmental and genetic effects.
}

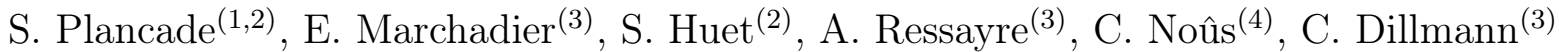

(1) University of Toulouse, INRAE, UR MIAT, F-31320, Castanet-Tolosan, France

(2) University Paris-Saclay, INRAE, MaIAGE, 78350, Jouy-en-Josas, France

(3) University Paris-Saclay, INRAE, CNRS, AgroParisTech, GQE - Le Moulon, 91190, Gif-sur-Yvette, France

(4) Cogitamus Laboratory, Castanet-Tolosan, France

Sandra Plancade: sandra.plancade@inrae.fr

Elodie Marchadier: elodie.marchadier@universite-paris-saclay.fr

Sylvie Huet: shuet.inra@gmail.com

Adrienne Ressayre: adrienne.ressayre@universite-paris-saclay.fr

Christine Dillmann: christine.dillmann@universite-paris-saclay.fr

\begin{abstract}
We propose a flexible statistical model for phyllochron that enables to seasonal variations analysis and hypothesis testing, and demonstrate its efficiency on a data set from a divergent selection experiment on maize.

The time between appearance of successive leaves or phyllochron enables to characterize the vegetative development of maize plants which determines their flowering time. Phyllochron is usually considered as constant over the development of a given plant, even though studies have demonstrated response of growth parameters to environmental variables. In this paper, we proposed a novel statistical approach for phyllochron analysis based on a stochastic process, which combines flexibility and a more accurate modelling than existing regression models. The model enables accurate estimation of the phyllochron associated with each leaf rank and enables hypothesis testing. We applied the model on an original maize dataset collected in fields from plants belonging to closely related genotypes originated from divergent selection experiments for flowering time conducted on two maize inbred lines. We showed that the main differences in phyllochron were not observed between selection populations (Early or Late), but rather ancestral lines, years of experimentation, and leaf ranks. Finally, we showed that phyllochron variations through seasons could be related to climate variations, even if the impact of each climatic variables individually was not clearly elucidated. All script and data can be found at https://doi.org/10.15454/CUEHO6
\end{abstract}

Date of submission: january 2021

\section{Introduction}

Annual plants produce new organs and undergo several developmental transitions almost all along their life-cycle. Hence in annual plant species, growth and development are critical to determine the time to completion of their life-cycle (from germination to seed maturation). The development schedule, and in particular the time of flowering that determines the onset of seed production within one season, needs to be synchronized with the season's length to maximise plant fitness or grain yield (Brachi et al., 2013). Plant development is characterized by successive events of organ production from (Poethig, 2003). Notably, apical meristems give rise to the roots and shoots. During vegetative growth, leaves are initiated from the Shoot Apical Meristem (SAM). After floral transition, the SAM becomes a male inflorescence that will later produce the panicule during the flowering phase and the total number of leaves is fixed (Hill and Li, 2016). Thus, the flowering time is tightly dependent on the duration of the vegetative phase, which is determined by the number of leaves to initiate from the meristem and by their rate of initiation (Padilla and Otegui, 
2005). Maize (Zea mays) is a monocotyledon species that initiates between seven to more than 18 phytomers before flowering (Flint-Garcia et al., 2005; Vidal and Andrieu, 2019). Observation of the time between successive leaves initiation (plastochron) requires destructive measurements and cannot be repeatedly performed on individual plants. However, the time between leaf appearance (phyllochron) enables to characterize the whole vegetative development of individual plants by avoiding plant dissection.

Environmental temperature is the climatic variable influencing the most plant development. Indeed, unlike homeotherms animals, plants do not maintain their temperature constant. Because of the high sensitivity of enzymatic reaction rates to temperature changes (Johnson et al., 1942), cell division rate can be considerably reduced (or stopped) in non optimal conditions leading to changes in growth rate with temperature. To take into account thermal dependence of plant growth rate, it is possible to model temperature-compensated developmental rates by measuring time in degree days (accumulated thermal time or ATT) taking into account species specific relationship between growth rates and daily temperatures. Notably, such approaches have been applied on maize grown in greenhouses, growth chambers and field conditions (Millet et al., 2019). This leads to a calibration of the equation of temperature-compensated rates of development when temperature fluctuates over a large range, for which thermal time based on a linear response is inefficient (Parent et al., 2010).

In addition to temperature, other climatic variables have been previously shown to modulate developmental rates in maize notably through alterations of cell's elongation rate. For instance, Granier and Tardieu (2009) demonstrated that water deficit results in a reduction of leaf length. Specifically to leaf appearance rates, a decrease of photosynthetically active radiation seems to slow down the phyllochron in a context of mixed cultivation systems (Zhu et al., 2014; Birch et al., 1998) while long photoperiods increase the leaf appearance rate (Warrington and Kanemasu, 1983). To a lesser extent, sowing date is another factor affecting leaf appearance rate (Birch et al., 1998), which can be considered as the resulting effect of multiple climatic variables.

Although these studies demonstrated changes in the phyllochron depending on the environmental conditions, phyllochron is usually considered as constant over the development of a given plant and characterized by the slope of linear regression between number of emerged leaves and thermal time (Padilla and Otegui, 2005). However, response of growth parameters and yield to within-season environmental variables have also been described (Chenu et al., 2008; Yu and Goh, 2019 ) and the impact of on-off climatic events on the phyllochron are worth to be considered and may question its constancy. A couple of phyllochron models with more flexible functions of ATT have been proposed, in particular bi- or tri-linear functions for rice (Clerget and Bueno, 2013) or splines for wheat (Baumont et al., 2019). Nevertheless, these models based on a regression between number of emerged leaves and thermal time ignore the statistical correlation between the number of leaves at successive time on the same plant (e.g. the number of leaves at time $t+d t$ is not smaller that the number of leaves at time $t$ ). Therefore, even if they are suitable for a descriptive analysis of phyllochron, the statistical analyses (confidence interval, test...) based on these models are biased. In this paper, we propose a flexible modelling of phyllochron based on thermal time which enables to release the classic linearity assumption. This model provides a more precise representation of phyllochron, but also enables to test for differences in phyllochron between various conditions and to evaluate the impact of climatic variables on variations of phyllochron throughout the season.

After domestication, maize was spread worldwide and adapted to a wide range of environmental conditions (Brandenburg et al., 2017). European and Northern american varieties lost sensitivity to photoperiod. Although similar response curves to temperature are observed for developmental processes of different lines (Parent and Tardieu, 2012), they still exhibit genetic variations for flowering time (Parent et al., 2018). These differences are generally associated with variation of the total leaf number: early varieties have a lower leaf number than late varieties (Li et al., 2016). However, flowering time may vary without changes in total leaf number (Durand et al., 2012), suggesting genetic variability for leaf appearance rate. Phyllochron genetic variability has been observed on maize inbred lines (Verheul et al., 1996) and hybrids (Padilla and Otegui, 2005). In the present study, we take advantage of maize genotypes exhibiting differences for flowering time to develop estimation method taking into account the experimental protocol of data collection.

We make use of an original plant material that resulted from twelve years of divergent selection for flowering time within single maize inbred lines under agronomical conditions (Durand et al., 2010, 2015). Within homogeneous genetic background, phenological shifts between Early and 
Late progenitors were selected, resulting in a difference reaching three weeks (150 degree days, (Durand et al., 2015)). Representative progenitors from generation G13 were chosen to monitor plant growth during three years, between 2014 and 2016. This study aims at evaluating the phyllochron differences between these genotypes and also to better understand whether and how climatic variables can lead to short term developmental responses.

\section{Materials and methods}

\subsection{Experimental plan/data collection}

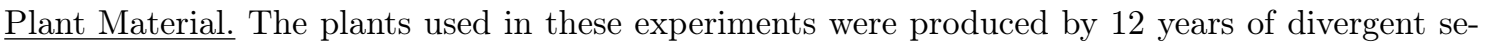
lection for flowering time in two maize inbred lines MBS847 (MBS) and F252. In each inbred, two early and two or three late genotypes were formed by recurrently selecting and selfing the earliest and lastest flowering plants in Early and Late selection populations respectively. In F252, early genotypes are named FE36 and FE39 and late ones FL27, FL317 and FL318. In MBS, early genotypes are named ME49 and ME52 and late ones ML40 and ML53. Details about of the selection and selfing processes are provided in Durand et al. (2010). In generation G13, contrasted flowering times were observed between Early and Late selection populations in both ancestral lines (Durand et al., 2015).

Crop experiment. G13 plants from the nine genotypes were sown on Saclay's Plateau (France) in 2014, 2015 and 2016. 25 seeds were sown in lines of $5.2 \mathrm{~m}$ with distance between two lines of $0.8 \mathrm{~m}$. For each inbred line, the parcel was formed of several plots of 14 lines protected on both sides by two border lines of control plants. In 2014, three lines were sown per genotype. In 2015, three to five lines were sown per genotype. In both years, genotypes were randomly assigned to lines and plots. In 2016, a particular attention was brought to ME52 and ML40 and 12 lines of each were sown in two plots. To protect plants from bird predation, experimental plots were protected with nettings installed immediately after sowing until most of the plants had at least six-seven visible leaves. Nettings were removed once when most plants had around three-four leaves, and the third leaf were marked using a pencil. From nettings removal to the emergence of panicule, the rank of every odd new leaf was marked with a pencil and the number of leaves of each plant counted twice a week (every two to four days). Temperature was recorded from the sowing date every hour at the climatic station hosted by our laboratory (station Gif-Sur-Yvette number 91272003 in the climatik INRAE database https://intranet.inrae.fr/climatik) and the mean temperature of the day was used to compute thermal time (Parent et al., 2010). A portion of the observed plants were dissected for further analysis, around a median leaf rank of 8.5 (year 2014), 10 (year 2015) and 9 (year 2016). These partially observed plants were used to infer the phyllochron model but their contribution is limited to the estimation of the first leaves appearance.

Altogether, we collected data on 1795 plants at various time points. Within each year $y$, observations consist in couples $\left(t_{y, l s g, p, j}, X_{y, l s g, p, j}\right)$, where $X_{y, l s g, p, j}$ is the leaf rank of the youngest visible leaf of plant $p$ from genotype $l s g$, with $l$ the ancestral line (F252 or MBS) and $s$ the selection population (Early or Late), observed at the $j^{\text {th }}$ observation time $t_{y, l s g, p, j}$. For each plant, the appearance of the two last leaves was not modelled and the corresponding records were removed. Moreover, plants with non-increasing numbers of leaves (error of measurement) were deleted. In 2014 we obtained data on the full phyllochron for 318 plants with 8-17 [mean 12.5] observation time points. In 2015, we obtained data on the full phyllochron for 371 plants with 8-21 [mean 13] time points, and partial measurements for 328 plants. In 2016 , we obtained data on the full phyllochron for 196 plants with 6-15 [mean 11.2] time points, and partial measurements for 233 plants.

\subsection{Statistical model for the phyllochron}

Phyllochron model was implemented separately for each year of experiment, thus parameters depend on the year but to simplify notations, reference to the year is omitted in this section.

Probabilistic model for the phyllochron. Denote by $Y_{l s g, p, f}$ the unobserved time interval between appearance of leaves of rank $f-1$ and $f$ on plant $p$ from genotype $l s g$. The phyllochron 
process of plant $p$ is characterised by the vector $\left(Y_{l s g, p, f}\right)_{f=1, \ldots, F^{l s g}}$ with $F^{l s g}$ be the overall maximum number of leaves for genotype $l s g$. Then, the time of appearance of leaf $f$ on plant $p$ from genotype $l s g$ is

$$
H_{l s g, p, f}=\sum_{f^{\prime}=1}^{f} Y_{l s g, p, f^{\prime}}
$$

We assume that the time interval between successive leaves $Y_{l s g, p, f}$ depends on some characteristics of the plant genotype $l s g$ and on the leaf rank $f=1, \ldots, F^{l s g}$ :

$$
Y_{l s g, p, f}=\mu_{l s g, f}+\text { plant level variation }
$$

Thus, the average phyllochron of genotype $l s g$ is characterized by the vector $\mu_{l s g}=\left(\mu_{l s g, 1}, \ldots \mu_{l s g, F^{l s g}}\right)$, and $\bar{\mu}_{l s g, f}=\sum_{f^{\prime}=1}^{f} \mu_{l s g, f^{\prime}}$ corresponds to the average time of appearance of leaf $f$. Note that no parametric form is assumed for the average phyllochron. Moreover, time intervals between appearance of successive leaves on each plant are assumed to be independent random variables and Gaussian distributed, thus

$$
\mathbf{Y}_{l s g, p}=\left(Y_{l s g, p, 1}, \ldots, Y_{l s g, p, F^{l s g}}\right) \sim \mathcal{N}_{F^{l s g}}\left(\mu_{l s g}, \Sigma_{l s g}\right)
$$

with $\Sigma_{l s g}=\operatorname{diag}\left(\sigma_{l s g, 1}^{2}, \ldots, \sigma_{l s g, F^{l s g}}^{2}\right)$ the diagonal variance-covariance matrix.

Note that the times $\left(H_{l s g, p, 1}, \ldots, H_{l s g, p, F^{l s g}}\right)$ of leaf appearance on a plant $p$ are not independent, as they result from accumulation of independent intervals between leaves $\left(Y_{l s g, p, f}\right)_{f}$, as illustrated on Figure 1-A.

Taking into account experimental constraints. Observations in the field started at a fixed time point, resulting in variations of the first leaf rank observed between plants. For each genotype $l s g$, we modelled the phyllochron on the range of leaf ranks $\left[f_{\min }^{l s g}, f_{\max }^{l s g}\right]$ such that leaf ranks $f_{\min }^{l s g}-1$ and $f_{\max }^{l s g}$ were observed on at least 10 plants per genotypes. Figure 1-B illustrates the observations available for a single plant, as compared to the full dynamics of leaf appearance.

Statistical model. First leaves being unobserved, we estimated the distribution of the time interval $H_{l s g, p, f_{\min }^{l s g}}$ between sowing and appearance of leaf of rank $f_{\min }^{l s g}$, denoted by cumulated phyllochron, and of the vector $\left(Y_{l s g, p, f}\right)_{f=f_{\min }^{l s g}+1, \ldots, f_{\max }^{l s g}}$ denoted by instant phyllochron. According to the probabilistic model $(2), H_{l s g, p, f_{\min }^{l s g}} \sim \mathcal{N}\left(\mu_{l s g}^{C}, \sigma_{l s g}^{C}\right)$, with

$$
\mu_{l s g}^{C}=\bar{\mu}_{l s g, f_{\min }^{l s g}}=\sum_{f^{\prime}=1}^{f_{\text {min }}^{l s g}} \mu_{l s g, f^{\prime}}, \quad \sigma_{l s g}^{C}=\sqrt{\sum_{f^{\prime}=1}^{f_{\min }^{l s g}} \sigma_{l s g, f^{\prime}}^{2}}
$$

$\left(\mu_{l s g}^{C}, \mu_{l s g, f}\right)$ are the main parameters of interest, while $\left(\sigma_{l s g}^{C}, \sigma_{l s g, f}\right)$ may be considered as nuisance parameters. For each genotype, parameters $\left(\mu_{l s g}^{C}, \sigma_{l s g}^{C},\left(\mu_{l s g, f}, \sigma_{l s g, f}\right)_{f=f_{\min }^{l s g}+1, \ldots, f_{\max }^{l s g}}\right)$ were estimated via a Monte Carlo Expectation Maximisation algorithm (Figure 2 and Suppl.Mat. A).

\subsection{Model comparisons}

Test genotypic groups effects. We made use of the hierarchical grouping of plants to understand the genetic factors that impact phyllochron. Indeed, differences between plants may come from ancestral lines (F252 versus MBS), selection population (Early versus Late), or genotypes within a selection population and an ancestral line. Note that because of the hierarchical structure of the models, significant differences between genotypes may result in significant differences between selection populations, independently of a divergent selection effect. In addition, statistical differences can occure either before the first observations and target the $\mu^{C}$ parameter, or on a the modeled leaf rank and target the $\mu$ parameters. Altogether, we ran seven different models $M_{i, j}$ (Table 1) implemented on the leaf ranks common to all genotypes: $\left[f_{\min }, f_{\max }\right]=\bigcap\left[f_{\min }^{l s g}, f_{\max }^{l s g}\right]$; indexes $i$ and $j$ denote the level of dependence of cumulated and instant phyllochron respectively, and values $0,1,2,3$ indicates that phyllochron is identical accross all plants (0), within the ancestral line (1), within the selection population (2) or within a genotype (3). 
Model $M_{00}$ assumes that all plants have the same phyllochron distribution whatever the ancestral line, the selection population or the genotype. Models $M_{10}$ and $M_{11}$ suppose that phyllochron varies with the ancestral line for at least one leaf rank, either during early developmental stages $\left(M_{10}\right)$ or anywhere throughout the season $\left(M_{11}\right) ; M_{11}$ is preferred to $M_{10}$ when the phyllochron differences occur on observed leaf ranks. Similarly, models $M_{21}$ and $M_{22}$ assume differences in phyllochron between Early and Late populations for at least one leaf rank, while models $M_{32}$ and $M_{33}$ assume differences between genotypes.

As phyllochron estimates highlight obvious differences between years of experimentation, a separate analysis was run for each year. Also, comparisons between $M_{12} / M_{22}$ and $M_{11}$ were performed either by pooling the two ancestral lines, or within each ancestral line. Similarly for comparisons between $M_{23} / M_{33}$ and $M_{22}$ within ancestral line and selection population.

Parametric sub-models of the phyllochron dynamics. In order to elucidate trends in the departure from the classic constant leaf rate appearance model, we considered parametric models for the instant phyllochron as a function of leaf rank, that can capture general trends by summarizing the vector $\left(\mu_{l s g, f_{\min }^{l s g}+1}, \ldots \mu_{l s g, f_{\max }^{l s g}}\right)$ through a reduced set of parameters for each genotype. We considered four parametric models. (i) The constant model supposes that phyllochron is constant throughout the season. (ii) The constant-rate model supposes a constant increase or decrease of phyllochron with leaf rank. (iii) The piecewise constant model allows a single change in the phyllochron at a given leaf stage. (iv) The piecewise linear model allows two increasing or decreasing phase. Details on model selection in Suppl. Mat. B.

Criteria for model comparison. We considered the $\chi^{2}$-likelihood ratio test, as well as the AIC and BIC criteria (Suppl. Mat. D). As these criteria rely on asymptotic considerations and may be biased in the context of a finite sample size, we validated them by a complementary permutation test. The instant phyllochrons of genotypes FE36 and FE39 on year 2015 were compared, with the null assumption of equal phyllochron distribution for the two genotypes. Then the same comparison was repeatedly performed while permutating the genotype labels and thus defining false genotypes; if the likelihood ratio test was unbiased, the p-value for the "false genotypes" would have been uniformly distributed on [0,1]; moreover, the most relevant criterion among AIC and BIC should have mostly selected the model under the null assumption.

Principal Components Analysis (PCA)

PCA was implemented on the set of vectors $\left(\widehat{\bar{\mu}}_{y, l s g, 8}, \widehat{\mu}_{y, l s g, 9}, \ldots, \widehat{\mu}_{y, l s g, 14}\right)$ for all combination of genotype $l s g$ and all year $y$. For genotypes FE36 and FL317 in $2014, f_{\max }^{y, l s g}=13$ so $\widehat{\mu}_{y, l s g, 14}$ was not estimated, and we replaced these two missing values by the average of $\widehat{\mu}_{y, l s g, 14}$ over all other genotype-year combinations.

\subsection{Impact of climate on phyllochron}

In order to elucidate the impact of climate on the variations of phyllochron throughout the season and between years, we considered a model where the interval times between successive leaves were regressed over climatic variables. First of all, a set of climatic variables recorded through the maize growth season were extracted from meteo station data. Secondly, a subset of variables associated with phyllochron variations was selected. Finally, the optimal time windows on which climate variables impact leaf appearance were selected.

\subsubsection{Extraction of representative climatic variables}

Thirty-one climatic variables were downloaded from the climatik INRAE database. Pearson correlation coefficient $r$ was calculated between each pair of climatic variables, and the distance between pairs of variables was defined as $1-r$, forming a distance matrix. Then, the Ward clustering algorithm was implemented on this distance matrix using the euclidean distance, leading to six clusters of climatic variables (Figure S3). Finally, a set $\mathcal{C}^{0}$ of six climatic variables typical of each cluster and easy to interpret was selected based on background knowledge:

- PAR: daily average photosynthetically active radiation (in Joules per cm2) 
- RF: rain falls (in millimeters per day)

- H: daily average atmospheric humidity (in \%)

- W: daily average wind speed (in meter per seconde)

- DT: dewpoint temperature $\left(\right.$ in $\left.{ }^{\circ} \mathrm{C}\right)$

- T: daily average temperature $\left(\right.$ in $\left.{ }^{\circ} \mathrm{C}\right)$

\subsubsection{Statistical model of climatic variables and phyllochron.}

We defined a model in which the average time intervals between successive leaves $\left(\mu_{y, l s g, f}\right)_{y, l s g, f}$ are explained by a set $\mathcal{C}$ climatic variables, instead of leaf ranks. More precisely, for year $y$, genotype $l s g$ and leaf rank $f$,

$$
m_{1}(\mathcal{C}, \Delta): \quad \mu_{y, l s g, f}=\alpha_{y, l}+\sum_{j \in \mathcal{C}} \beta_{y, l, j} C_{y, l s g, f, j}^{\left(\Delta_{j}\right)}+A_{l s g}+\varepsilon_{y, l s g, f}
$$

with $C_{y, l s g, f, j}^{\left(\Delta_{j}\right)}$ the average value of the $j^{\text {th }}$ climatic variable on a $\Delta_{j}$-day window before the time of appearance $\bar{\mu}_{y, l s g, f}$ of leaf $f$, transformed into calendar time (Figure S4). The line-year effect $\alpha_{y, l}$ accounts for the potential effect of genetic background interacting with the year of experiment (climatic and cultivation conditions and sowing date). The effect of each climatic variable $j \in \mathcal{C}$ depends on the year-line background through the coefficient $\beta_{y, l, j}$. Moreover, the effect of genotype is accounted for through a Gaussian random effect $A_{l s g}$ identical over years, and residuals $\varepsilon_{y, l s g, f}$ are assumed i.i.d. Gaussian centered.

The associated null model supposes that leaf appearance rate is constant over the season for each genotype:

$$
m_{0}: \mu_{y, l s g, f}=\alpha_{y, l}+A_{l s g}+\varepsilon_{y, l s g, f}
$$

Models $\left(m_{1}\right)$ and $\left(m_{0}\right)$ are defined for the true average interval time between leaves $\left(\mu_{y, l s g, f}\right)$, but they were inferred based on estimates.

\subsubsection{Selection of the climatic variables that impact phyllochron.}

A set $\mathcal{C}^{\text {select }}$ of climatic variables was extracted by a backward stepwise selection based on the AIC criterion. Starting from the reference model $m_{1}^{\text {full }}=m_{1}\left(\mathcal{C}^{0},(10, \ldots, 10)\right)$ including the six climatic variables computed on a fixed 10-day time window, at each step the variable whose removal lead to the strongest decrease of AIC was eliminated; the procedure stopped when AIC did not decrease anymore. The selected model is $m_{1}\left(\mathcal{C}^{\text {select }},(10, \ldots, 10)\right)$.

\subsubsection{Determination of the time window}

Climatic variables may have either a short-time or a long-term effect on phyllochron, governed by the window $\Delta_{j}$ which is assumed to depend on the climatic variable but to be identical through genotypes and year and throughout the season. The vector of optimal time windows $\Delta^{\text {opt }}$ was chosen by AIC criterion over all combinations of windows $\Delta \in\{1 ; 2 ; 3 ; 5 ; 10 ; 15 ; 20 ; 25\}^{\# \mathcal{C}^{\text {select }}}$ with $\# \mathcal{C}^{\text {select }}$ the number of variables in $\mathcal{C}^{\text {select }}$. The model with selected climate variables and optimal time windows is denoted $m_{1}^{\text {best }}=m_{1}\left(\mathcal{C}^{\text {select }}, \Delta^{\text {opt }}\right)$.

\section{Results}

\subsection{Total leaf number}

Figure S1 displays the distribution of the total leaf number. Early genotypes have globally less leaves than Late genotypes from the same genetic background, and this difference is stronger for the ancestral line MBS with 16-18 leaves versus 18-20 leaves. Besides, the total leaf number is similar across years within genotypes, even slightly higher in 2014 than in 2015, and in 2015 than in 2016 except for genotype FL317. By construction, the maximum modelled leaf rank $f_{\max }^{y, l s g}$ is correlated to the total leaf number, thus the trends observed on the latter are recovered on the former. 


\subsection{Phyllochron estimates by genotypes}

Figure 3 displays the estimates of the cumulated phyllochron between sowing and the appearance of the $8^{\text {th }}$ leaf, equal to $\widehat{\mu}_{y, l s g}^{C}$ if $f_{\min }^{y, l s g}=8$ and $\widehat{\mu}_{y, l s g}^{C}+\widehat{\mu}_{y, l s g, 8}$ if $f_{\min }^{y, l s}=7$, as well as the interval of time between leaves 8 and 13

$$
\sum_{f^{\prime}=9}^{13} \widehat{\mu}_{y, l s g, f^{\prime}}
$$

The cumulated phyllochron (Figure 3A) is mainly associated with year, being $3-5$ degree days shorter in 2015 than in 2014 and 2016, and is shorter in ancestral line F252 than MBS. Contrary to the cumulated phyllochron, the instant phyllochron (Figure 3B) is shorter in 2014 than in 2015, and intermediate in 2016. Difference between years is more pronounced on ancestral line MBS (difference of 3-4 degree days) than on ancestral line F252 (1-2 degree days). Within selection populations, differences between genotypes are mostly preserved from one year to the other for both cumulated and instant phyllochrons. Note two exceptions: for groups F252-Early (cumulated phyllochron) and MBS-Early (instant phyllochron).

More in details, the temporal trend of the instant phyllochron strongly varies between years (Figure 4). In 2014 and 2016, time interval between successive leaves varies moderately between 3 and 5 degree days, except an increasing trend between leaves 13 and 14 in F252 genotypes, and is globally shorter in 2014 than in 2016 (3.5 versus 4 degree days on average). In 2015, the phyllochron shows stronger variations throughout the season, between 3 and 7 degree days, with an increasing and a decreasing phase for MBS genotypes, and a rather constant increase for F252 genotypes.

\subsection{Model comparisons}

Statistical tests. Genotypic groups comparisons were performed only for years 2014 and 2015, where all genotypes were observed, and were restricted to the range of leaf ranks $[9,13]$, common to all genotypes. The preliminary permutation test indicated that $\chi^{2}$-test and AIC criterion were reliable for group effect testing, even if AIC was slightly biased in favor of the largest model (Suppl. Mat. D).

Table 2 displays the result of comparisons between ancestral line, selection and genotypes. Both criteria mostly provide coherent conclusions, while AIC is slightly less conservative as highlighted by the permutation test. Effects were analysed based on a $0.01 \mathrm{p}$-value threshold.

The results indicate a strong effect of the ancestral line on both cumulated and instant phyllochron. Difference between selection populations depends on year and ancestral line; in 2014 a significant difference was observed on the instant phyllochron but not on the cumulated phyllochron for the general comparison between Early and Late selection populations, and between Early and Late populations in F252, while ancestral line MBS displays a selection population effect. In 2015, we found a difference between selection population on instant phyllochron in both F252 and MBS backgrounds, but the difference on cumulative phyllochron was only significant for MBS. Finally, we find a significant genotypic effect on both the instant and the cumulative phyllochron except for the Early MBS population in 2014 (no effect), and the Early F252 population in 2015 (no cumulative effect).

Graphical analysis. Results of statistical tests can be refined by graphical analysis of the estimates of instant (Figure 5) and cumulated (Figure 3A) phyllochrons.

In 2014, significant differences between ancestral lines are propelled by lower values of the cumulated phyllochron but higher values of instant phyllochron at leaf 13 and in F252 genotypes, as compared to MBS genotypes. Differences between Early and Late genotypes are only significant within F252 genotypes and concern the instant phyllochron, which tends to increase between leaf nine and leaf 13 in Late genotypes, and to stay constant in Early genotypes.

In 2015, significant differences between ancestral lines are propelled by lower values of the cumulated phyllochron and higher values of the instant phyllochron at leaf 13 in F252 genotypes, but also by higher values of the instant phyllochron at leaf 11 and leaf 12 in MBS genotypes. Significant differences between selection populations for the instant phyllochron are probably due to lower values at leaf nine in Late F252 as compared to Early F252, and to higher values at leaf 11 in Early MBS as compared to Late MBS.

Altogether, we found significant differences on phyllochron at all level of grouping. Differences between ancestral lines are particularly clear in 2015: instant phyllochron tends to increase with 
leaf rank in F252 genotypes, while it shows a maximum around leaf ranks 11-12 in MBS genotypes. Differences between genotypes were also significant at each leaf stage, except for Early MBS genotypes in 2014.

\subsection{Parametric models}

Figure 6 displays the best parametric sub-model for each genotype. Overall, the sub-models fit well the complete models, and manage to capture the seasonal tendencies. The constant phyllochron model was selected only for one genotype in 2014 (ME052). Moreover, the major differences previously observed are recovered by the best parametric sub-model. In 2014 and 2016, the variations of phyllochron are moderate with a few exceptions in 2014 for the Late F252 genotypes, that increases either linearly (FL317, FL318) or piecewise (FL027). In 2015, the phyllochron of most F252 genotypes increases throughout the season, while the phyllochron of MBS genotypes and FL027 shows a maximum between 11 and 13 leaves, with an increase before and a decrease after.

\subsection{Patterns of variation}

PCA of all phyllochron estimates $\left(\widehat{\mu}_{y, l s g, f}\right)_{y, l s g, f}$ (Figure 7 ) provides and overview of global patterns in phyllochron. The first axis (49\% of total inertia), discriminates year 2015 on the right versus years 2014 and 2016 on the left. The second axis (21\% of total inertia) discriminates ancestral lines: F252 genotypes mostly have negative coordinates, while MBS genotypes have positive coordinates. No clear discrimination was observed between Early and Late genotypes within each ancestral line. Other differences concern genotypes, independently of the year of observation, the ancestral line or the selection effect. Besides, the cumulated phyllchron as well as the instant phyllochron of leaves nine to 13 are loaded by both axes, and thus are associated with both ancestral line and year. On the contrary, $\widehat{\mu}_{14}$ is mostly associated with ancestral line. Finally, phyllochrons parameters are ordered clockwise by leaf ranks in the correlation circle, which indicates that intervals between successive leaves are similar for close leaf ranks.

\subsection{Input from climatic variables}

The AIC decreases strongly between the null model $m_{0}$ and the model $m_{1}^{\text {best }}$ (Table 3 ), thus the climatic model explains a significant part of phyllochron variations. Moreover, the model recovers seasonal trends for all genotypes and years, notably the clear seasonal trend in 2015, and the residuals are small and do not show any residual seasonal effect (Figure 8). Besides, variable selection seems to have a weaker impact than window selection, as indicated by AIC decrease (5.02 and 19.44 resp.)

More in details, the set $\mathcal{C}^{\text {select }}$ of selected variables associated with phyllochron variations includes : dewpoint temperature (DT), wind (W), humidity $(\mathrm{H})$ and radiation (PAR). The optimal time windows are respectively 20 days (DT), five days (W), 20 days (H), and five days (PAR). AIC globally increases as each time window moves away from its optimal value, all other windows being fixed to optimal values (Figure 9-top). Moreover, the optimality of each selected window is globally preserved when the other windows vary (Figure 9-bottom). Nevertheless, the 10 sets of windows leading to the lowest AIC are very different, which suggest that variations of one window can be compensated by variations of the other (Table 4). The sign of model coefficients, which should indicate if each climatic variable accelerates or slows down the leaf appearance process, varies with year and ancestral line (Table 5).

\section{Discussion}

\subsection{A stochastic process model for maize phyllochron}

In this paper, we proposed a flexible model for phyllochron based on a stochastic process in which phyllochron depends on leaf rank non-parametrically. Inference is far more complex than with the linear model or even with more flexible regression models, and requires a specific algorithm. This model is more flexible than the classic linear phyllochron model, which assumes a constant leaf appearance rate, and the results on our dataset indicate a strong departure from this assumption, notably in 2015, which validates a posteriori the relevance of a more general modelling, particularly 
in the context of peculiar climatic conditions. Our analysis of various parametric sub-models suggests that a two-phase model could represent a reasonable compromise between simplicity and flexibility, even if the complete model is statistically more accurate. Our model differs from the flexible regression models in literature (Clerget and Bueno, 2013; Baumont et al., 2019) in which variations of phyllochron throughout season are modelled on an external time scale (the thermal time), while in our model these variations are associated with the development of the plant through the leaf stage. Note that a given leaf rank can correspond to different periods of the season for different genotypes; one's has to keep in mind this aspect in the comparison between genotypes.

Furthermore, we proposed an unbiased statistical procedure to compare phyllochron in various conditions, that we applied to genotypic groups comparison. Generally, a price to pay for flexibility is the larger number of parameters, which requires more data to be correctly estimated, and this limitation is particularly true in the context of interval censoring with less informative observations. Nevertheless, our results indicate that aproximately 40 plants by genotypes (year 2014) were sufficient to enhance significant differences between genotypic groups resulting from a recent divergence.

The inference algorithm we proposed assumes Gaussian plant level variations around the genotype average phyllochron. Approximation of a positive variable by a Gaussian distribution is acceptable if the standard deviation is smaller than three-four times the mean, which is the case for most but not all genotypes on our data set. Nevertheless, Gaussian approximation is very common in statistical modelling, and it can be shown in diverse contexts that it leads to unbiased or moderately biased results.

The effect of climate variables on phyllochron was assessed with a two-step procedure: first, the parameters of the phyllochron model were inferred for each genotype and each year, then climate variables cumulated on window before appearance of each leaf were regressed on the average interval between leaves. This approach can be compared notably to genotype-to-phenotype studies in which a dynamic phenotype is summarised by a restricted set of parameters on which statistical analyses are performed (Reymond et al., 2004; Marchadier et al., 2019). Nevertheless the climate variable and windows selection via the two-step procedure does not account for precision in the estimation of phyllochron parameters. We are currently working on a more general framework based on semiMarkov models that can handle various distributions and thus relax normality assumption, and that would directly include longitudinal covariates such as climatic variables within the phyllochron model.

\subsection{Differences in phyllochron between closely related genotypes}

As previously published, maize lines produced by Saclay's DSE experiment exhibit a gradual flowering time divergence over the first 13 generations (Durand et al., 2015). The characterization of the phyllochron of these genotypes, as performed here, enables to better understand the developmental changes that could underlie such a response to selection. Unsurprisingly, we observed that the total leaf number was impacted during the selection process, with late populations tending to produce more leaves than early genotypes, (Durand et al., 2012) especially in the MBS genetic background. Apart from temporal variations of phyllochron, a simple change in leaf number would be sufficient to accelerate or delay the flowering time.

However, significant differences of phyllochron are observed between the DSE selection population and genotypes selected in response to the same selective pressure (earliness or lateness). These differences appear robust to environmental variations as they are mostly preserved from one year to the other for both the cumulated and the instant phyllochron. They could be explained by (i) random variations appearing through the selection process without contributing to the response to selection (genetic drift) and/or (ii) independent changes contributing to the adaptation to the selection pressure via different developmental mechanisms (i.e. convergent evolution). Nevertheless, no general trend could characterize Early and Late selection branchs; in particular, genotypes from the Early flowering selection branch do not display a faster development than the late one, which pleads for the first hypothesis.

\subsection{Phyllochron temporal trends and climate}

As different genotypes do not experience the same environment at the same developmental stage, differences in phyllochron observed between genotypes may originate either from selection or from differences in environment. We proposed a model that includes longitudinal climatic variables 
and thus enables a calendar time analysis, with selection of both climatic variables and timewindows preceding leaf appearance. As phyllochron temporal trends in 2015 strongly differ between ancestral lines with an increasing and a decreasing phase for MBS genotypes and two increasing phases for F252, potential interactions between climate and ancestral line and year were considered. Moreover, the climatic context that could result from unobserved environmental variables or model mispecification is incorporated through a coefficient depending on year and ancestral line.

After variables and time window selection, the climatic model includes four climatic variables, whose effects are added to the cumulative temperature taken into account in thermal time calculation. These variables enable to recover seasonal variations in phyllochron but the coefficient signs are not coherent between years and lines and the time window selection is unstable. Therefore interpretation of the results related to each variable would be hazardous.

Unstable results may originate either from (i) potential statistical biases of the two-step approach underlined in Section 4.1, (ii) correlations between climatic variables, (iii) simplicity of the model that can not recover complex behaviours. Notably, the model does not account for potential non-monotonous effect of variables (medium optimum value). Moreover, additive modelling of covariate effects may not be enough to recover complex interplays (Matiu et al., 2017). Nevertheless, a richer model would be at the price of a large number of parameters with respect to the number of phyllochron estimates used for model fitting, and could not be properly inferred with our limited number of genotypes.

In summary, the results suggest a strong impact of climate on phyllochron. Indeed, a limited set of variables enables to recover temporal trends, even if the climatic model was not sufficient to elucidate the impact of each variable. Besides, the phyllochron estimates display temporal trends mainly associated with year, notably successive leaves are alike for close leaf ranks, which suggests a medium or long term impact of climate on phyllochron.

\subsection{Conclusion}

Our phyllochron model based on a stochastic process enables to detect fine differences between related genotypes up to a moderate experimental effort (10 to 20 measurements throughout the season on 30-50 plants by genotypes). On the DSE dataset, we showed that the major sources of differences for the phyllochron were not the selection population (Early or Late), but rather the ancestral line (F252 or MBS), the year of experimentation, and the leaf rank. Moreover, our results clearly indicate that phyllochron is not constant throughout the season, and these temporal trends could be associated with climate.

\section{Data availability statement.}

All scripts and data can be found at https://doi.org/10.15454/CUEHO6

\section{References}

Baumont, M., B. Parent, L. Manceau, H. E. Brown, S. M. Driever, et al., 2019 Experimental and modeling evidence of carbon limitation of leaf appearance rate for spring and winter wheat. J Exp Bot 70: 2449-2462.

Birch, C. J., J. Vos, J. Kiniry, H. J. Bos, and A. Elings, 1998 Phyllochron responds to acclimation to temperature and irradiance in maize. Field Crops Research 59: 187-200.

Botev, Z. and L. Belzile, 2020 Truncatednormal: Truncated multivariate normal and student distributions $\mathrm{R}$ package version 2.2 .

Brachi, B., R. Villoutreix, N. Faure, N. HautekÃ “ete, Y. Piquot, et al., 2013 Investigation of the geographical scale of adaptive phenological variation and its underlying genetics in Arabidopsis thaliana. Molecular Ecology 22: 4222-4240.

Brandenburg, J.-T., T. Mary-Huard, G. Rigaill, S. J. Hearne, H. Corti, et al., 2017 Independent introductions and admixtures have contributed to adaptation of European maize and its American counterparts. PLoS genetics 13: e1006666. 
Chenu, K., S. C. Chapman, G. L. Hammer, G. McLean, H. B. H. Salah, et al., 2008 Shortterm responses of leaf growth rate to water deficit scale up to whole-plant and crop levels: an integrated modelling approach in maize. Plant, Cell \& Environment 31: 378-391.

Clerget, B. and C. S. Bueno, 2013 The effect of aerobic soil conditions, soil volume and sowing date on the development of four tropical rice varieties grown in the greenhouse. Functional Plant Biology 40: 79-88.

Durand, E., S. Bouchet, P. Bertin, A. Ressayre, P. Jamin, et al., 2012 Flowering time in maize: linkage and epistasis at a major effect locus. Genetics 190: 1547-1562.

Durand, E., M. I. Tenaillon, X. Raffoux, S. ThÃCpot, M. Falque, et al., 2015 Dearth of polymorphism associated with a sustained response to selection for flowering time in maize. BMC evolutionary biology 15: 103.

Durand, E., M. I. Tenaillon, C. Ridel, D. Coubriche, P. Jamin, et al., 2010 Standing variation and new mutations both contribute to a fast response to selection for flowering time in maize inbreds. BMC evolutionary biology 10: 2 .

Flint-Garcia, S. A., A.-C. Thuillet, J. Yu, G. Pressoir, S. M. Romero, et al., 2005 Maize association population: a high-resolution platform for quantitative trait locus dissection. The Plant Journal 44: $1054-1064$.

Granier, C. and F. Tardieu, 2009 Multi-scale phenotyping of leaf expansion in response to environmental changes: the whole is more than the sum of parts. Plant, Cell \& Environment 32: $1175-1184$.

Hill, C. B. and C. Li, 2016 Genetic Architecture of Flowering Phenology in Cereals and Opportunities for Crop Improvement. Frontiers in Plant Science 7.

Johnson, F. H., H. Eyring, and R. W. Williams, 1942 The nature of enzyme inhibitions in bacterial luminescence: Sulfanilamide, urethane, temperature and pressure. Journal of Cellular and Comparative Physiology 20: 247-268.

Li, D., X. Wang, X. Zhang, Q. Chen, G. Xu, et al., 2016 The genetic architecture of leaf number and its genetic relationship to flowering time in maize. The New Phytologist 210: 256-268.

Marchadier, E., M. Hanemian, S. Tisné, L. Bach, C. Bazakos, et al., 2019 The complex genetic architecture of shoot growth natural variation in arabidopsis thaliana. PLOS Genetics 15: 1-27.

Matiu, M., D. P. Ankerst, and A. Menzel, 2017 Interactions between temperature and drought in global and regional crop yield variability during 1961-2014. PLOS ONE 12: 1-23.

Millet, E. J., W. Kruijer, A. Coupel-Ledru, S. Alvarez Prado, L. Cabrera-Bosquet, et al., 2019 Genomic prediction of maize yield across European environmental conditions. Nature Genetics 51: $952-956$.

Padilla, J. M. and M. E. Otegui, 2005 Co-ordination between leaf initiation and leaf appearance in field-grown maize (Zea mays): genotypic differences in response of rates to temperature. Annals of Botany 96: 997-1007.

Parent, B., M. Leclere, S. Lacube, M. A. Semenov, C. Welcker, et al., 2018 Maize yields over Europe may increase in spite of climate change, with an appropriate use of the genetic variability of flowering time. Proceedings of the National Academy of Sciences of the United States of America 115: 10642-10647.

Parent, B. and F. Tardieu, 2012 Temperature responses of developmental processes have not been affected by breeding in different ecological areas for 17 crop species. The New Phytologist 194: $760-774$.

Parent, B., O. Turc, Y. Gibon, M. Stitt, and F. Tardieu, 2010 Modelling temperature-compensated physiological rates, based on the co-ordination of responses to temperature of developmental processes. Journal of Experimental Botany 61: 2057-2069. 
Poethig, R. S., 2003 Phase change and the regulation of developmental timing in plants. Science 301: $334-336$.

Reymond, M., B. Muller, and F. Tardieu, 2004 Dealing with the genotype $\times$ environment interaction via a modelling approach: a comparison of QTLs of maize leaf length or width with QTLs of model parameters. Journal of Experimental Botany 55: 2461-2472.

Verheul, M., C. Picatto, and P. Stamp, 1996 Growth and development of maize (zea mays 1.) seedlings under chilling conditions in the field. European Journal of Agronomy 5: $31-43$.

Vidal, T. and B. Andrieu, 2019 Contrasting phenotypes emerging from stable rules: A model based on self-regulated control loops captures the dynamics of shoot extension in contrasting maize phenotypes. Annals of Botany .

Warrington, I. J. and E. T. Kanemasu, 1983 Corn growth response to temperature and photoperiod i. seedling emergence, tassel initiation, and anthesis1. Agronomy Journal 75: 749-754.

Yu, J. and G. Goh, 2019 Estimating Non-Additive Within-Season Temperature Effects on Maize Yields Using Bayesian Approaches.

Zhu, J., J. Vos, W. van der Werf, P. E. L. van der Putten, and J. B. Evers, 2014 Early competition shapes maize whole-plant development in mixed stands. Journal of Experimental Botany 65: $641-653$.

Zoppé, A., Y.-P. A. Buu, and B. Flury, 2001 Parameter estimation under constraints for multivariate normal distributions with incomplete data. Journal of Educational and Behavioral Statistics 26: $219-232$.

\section{Tables}

\begin{tabular}{lrrrll}
\hline Model & Cumulated phyllochron parameters & Instant phyllochron parameters & Model name \\
\hline$M_{00}$ & $\forall(l, s, g), \quad\left(\mu_{l s g}^{C}, \sigma_{l s g}^{C}\right)=\left(\mu^{C}, \sigma^{C}\right)$ & $\forall(l, s, g), \quad\left(\mu_{l s g}, \sigma_{l s g}\right)=(\mu, \sigma)$ & identical \\
$M_{10}$ & $\forall(s, g), \quad\left(\mu_{l s g}^{C}, \sigma_{l s g}^{C}\right)=\left(\mu_{l}^{C}, \sigma_{l}^{C}\right)$ & $\forall(l, s, g), \quad\left(\mu_{l s g}, \sigma_{l s g}\right)=(\mu, \sigma)$ & C-line \\
$M_{11}$ & $\forall(s, g), \quad\left(\mu_{l s g}^{C}, \sigma_{l s g}^{C}\right)=\left(\mu_{l}^{C}, \sigma_{l}^{C}\right)$ & $\forall(s, g)$, & $\left(\mu_{l s g}, \sigma_{l s g}\right)=\left(\mu_{l}, \sigma_{l}\right)$ & $(\mathrm{C}+\mathrm{I})$-line \\
$M_{21}$ & $\forall g, \quad\left(\mu_{l s g}^{C}, \sigma_{l s g}^{C}\right)=\left(\mu_{l s}^{C}, \sigma_{l s}^{C}\right)$ & $\forall(s, g), \quad\left(\mu_{l s g}, \sigma_{l s g}\right)=\left(\mu_{l}, \sigma_{l}\right)$ & C-selection \\
$M_{22}$ & $\forall g, \quad\left(\mu_{l s g}^{C}, \sigma_{l s g}^{C}\right)=\left(\mu_{l s}^{C}, \sigma_{l s}^{C}\right)$ & $\forall g, \quad\left(\mu_{l s g}, \sigma_{l s g}\right)=\left(\mu_{l s}, \sigma_{l s}\right)$ & $(\mathrm{C}+\mathrm{I})$-selection \\
$M_{32}$ & $\quad\left(\mu_{l s g}^{C}, \sigma_{l s g}^{C}\right)=\left(\mu_{l s g}^{C}, \sigma_{l s g}^{C}\right)$ & $\forall g, \quad\left(\mu_{l s g}, \sigma_{l s g}\right)=\left(\mu_{l s}, \sigma_{l s}\right)$ & C-genotype \\
$M_{33}$ & $\left(\mu_{l s g}^{C}, \sigma_{l s g}^{C}\right)=\left(\mu_{l s g}^{C}, \sigma_{l s g}^{C}\right)$ & $\left(\mu_{l s g}, \sigma_{l s g}\right)=\left(\mu_{l s g}, \sigma_{l s g}\right)$ & $(\mathrm{C}+\mathrm{I})$-genotype \\
\hline
\end{tabular}

Table 1: Models for genotypic groups effects. C: cumulated phyllochron, I: instant phyllochron.

\begin{tabular}{|c|cc|}
\hline Model & number of parameters & AIC \\
\hline$m_{0}$ & 7 & 395.72 \\
\hline$m_{1}^{\text {full }}=m_{1}\left(\mathcal{C}^{0},(10, \ldots, 10)\right)$ & 37 & 267.52 \\
\hline$m_{1}=m_{1}\left(\mathcal{C}^{\text {select }},(10, \ldots, 10)\right.$ & 27 & 262.50 \\
\hline$m_{1}^{\text {best }}=m_{1}\left(\mathcal{C}^{\text {select }}, \Delta^{\text {opt }}\right)$ & 31 & 243.06 \\
\hline
\end{tabular}

Table 3: Comparison of climatic models. $m 0$ : no effect of climatic variables; $m_{1}^{f u l l}:$ model with the six climatic variables and a time window of 10 days for all variables; $m_{1}\left(\mathcal{C}^{\text {select }},(10, \ldots, 10)\right)$ model with the 4 selected climate variables and a time window of 10 days for all variables; $m_{1}^{\text {best }}$ : model with the 4 selected climate variables and the optimal time windows. 


\begin{tabular}{|c|c|c|c|c|}
\hline DT & PAR & W & H & AIC \\
\hline 20.00 & 5.00 & 5.00 & 20.00 & 235.07 \\
2.00 & 25.00 & 15.00 & 20.00 & 235.40 \\
3.00 & 25.00 & 15.00 & 20.00 & 235.80 \\
20.00 & 10.00 & 5.00 & 10.00 & 235.87 \\
3.00 & 10.00 & 5.00 & 10.00 & 236.78 \\
20.00 & 10.00 & 5.00 & 20.00 & 236.84 \\
1.00 & 25.00 & 15.00 & 20.00 & 237.61 \\
3.00 & 10.00 & 5.00 & 20.00 & 238.04 \\
4.00 & 10.00 & 5.00 & 20.00 & 238.68 \\
15.00 & 10.00 & 5.00 & 10.00 & 239.12 \\
\hline
\end{tabular}

Table 4: Combinations of time windows leading to the smallest AIC value in model $m_{1}\left(\mathcal{C}^{\text {select }}, \Delta\right)$, with the set of selected variables: Dewpoint Temperature (DT), Wind (W), Humidity (H), Photosynthetically Active Radiation (PAR). The first line corresponds to the optimal time windows of the $m_{1}^{\text {best }}$ model.

\begin{tabular}{|c|c|c|c|c|c|}
\hline & F252-2014 & F252-2015 & MBS-2014 & MBS-2015 & MBS-2016 \\
\hline$D T(20$ days $)$ & -12.89 & 6.19 & -4.95 & 5.09 & -1.42 \\
\hline$P A R(5$ days $)$ & 0.94 & -0.17 & 1.99 & 5.28 & -1.89 \\
\hline$W(5$ days $)$ & -1.36 & -2.69 & 1.13 & -3.38 & -1.47 \\
\hline$H(20$ days $)$ & 31.08 & -10.10 & 9.75 & 9.90 & -0.22 \\
\hline
\end{tabular}

Table 5: Coefficients of the $m_{1}^{\text {best }}$ model. 

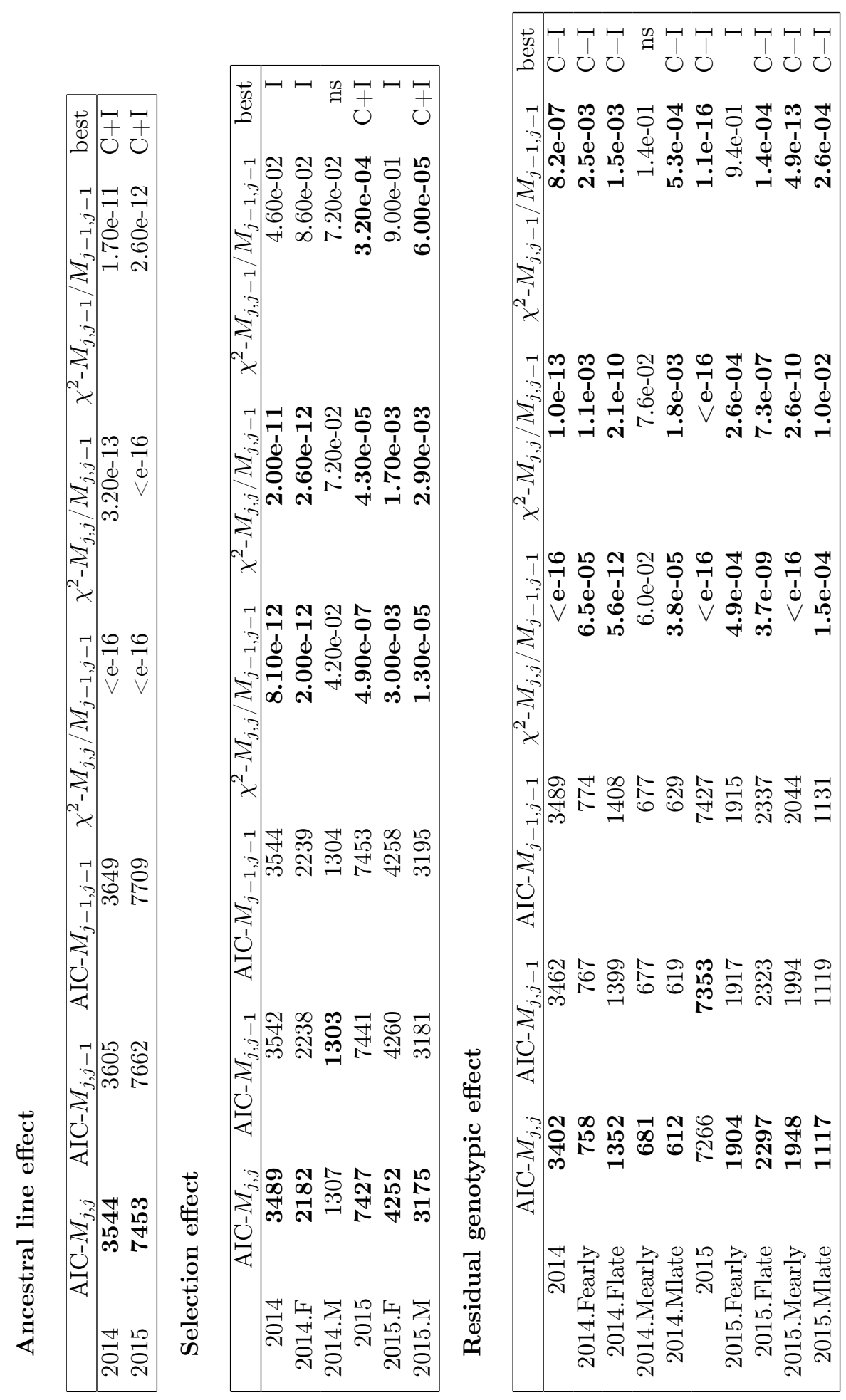


\section{Figures}

\section{A. Random process}

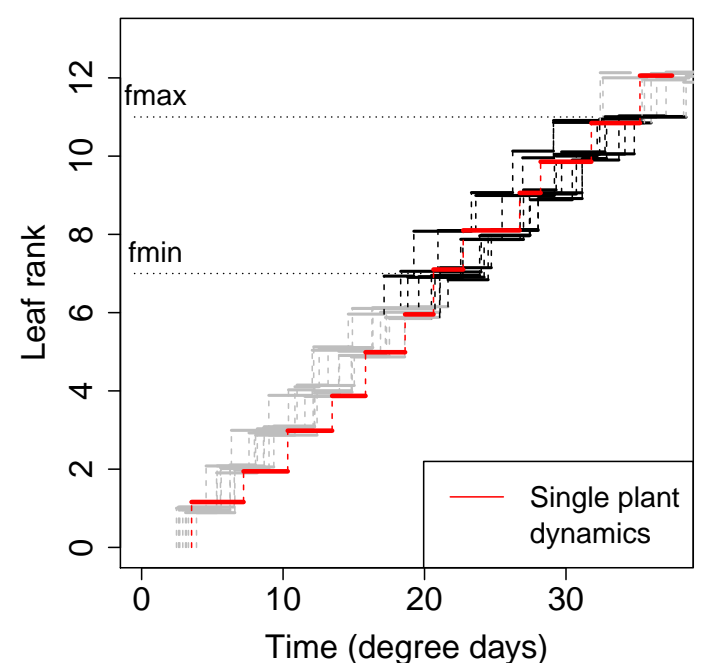

B. Process and observations for one plant

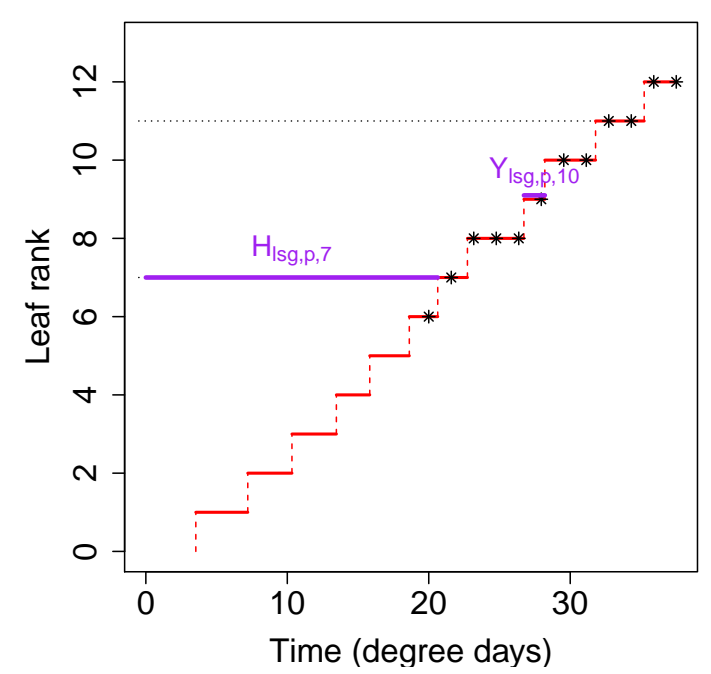

Figure 1: Illustration of the phyllochron process. The leaf appearance process of a plant is characterised by the number of visible leaves at each time (in degree-days), or equivalently by the time interval between successive leaves. For a plant $p$ and a leaf rank $f$, the horizontal bar with y-coordinate equal to $f-1$ corresponds to the time interval $Y_{l s g, p, f}$ between appearance of leaves $f-1$ and $f$. A. Leaf appearance dynamics for several plants from the same genotype. $\left[f_{\min }, f_{\max }\right]$ correspond to the interval on which the phyllochron is estimated; Grey lines correspond to leaf stages that are not modelled. The dynamic of a single plant is highlighted in red. $\mathbf{B}$. Representation of the dynamics and the observations of a single plant. $H_{l s g, p, f}$ is the time of appearance of leaf $f$ since sowing. Dots represent the observation time points, at which the number of visible leaves is monitored

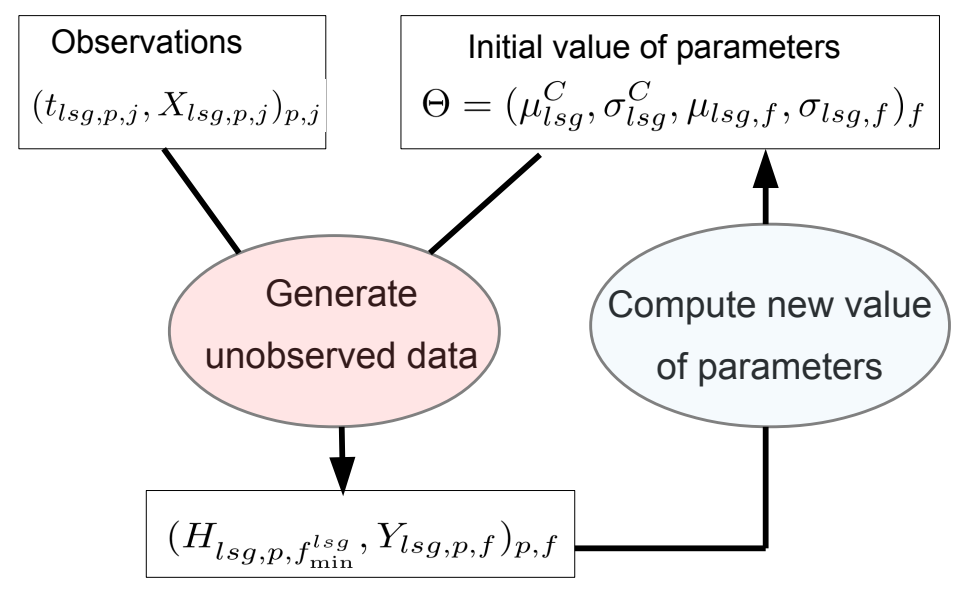

Figure 2: Monte Carlo Expectation Maximisation algorithm. Starting from an initial value of the parameters, the unobserved times of leaf appearance are drawn from their distribution given the observed data. Then, the maximum likelihood estimator is inferred from the simulated unobserved data, producing a new estimate of the parameters. The algorithm is iterated until stabilisation of the parameters. 


\section{A. Cumulated phyllochron until leaf 8}

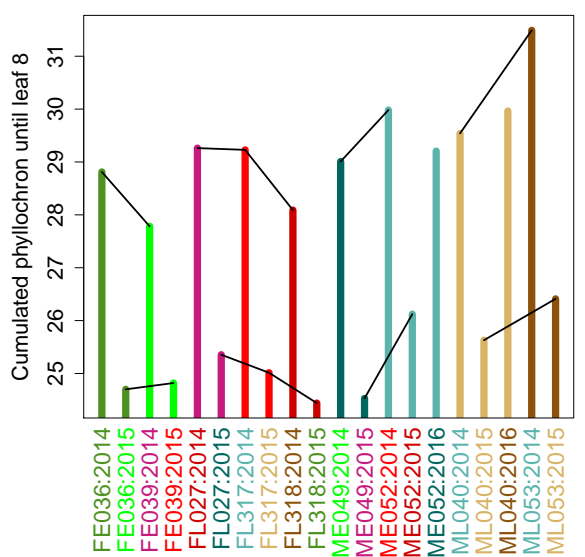

B. Time between leaves 8 and 13

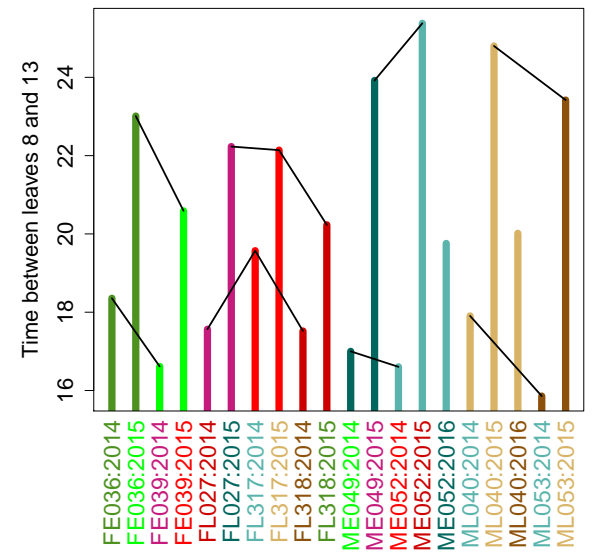

Figure 3: Cumulated and instant phyllochron. A. Time in degree days to reach eight visible leaves. B. Time interval in degree days between appearance of leaves eight and 13. Straight lines join genotypes from the same selection population observed the same year.

2014

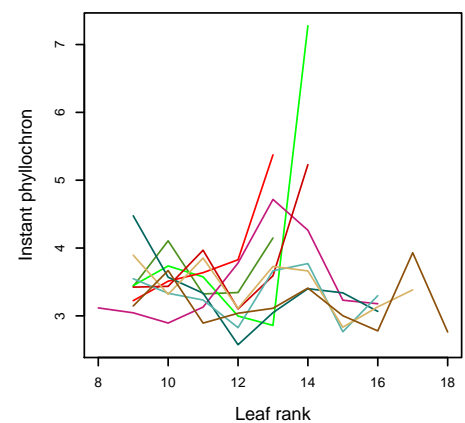

2015

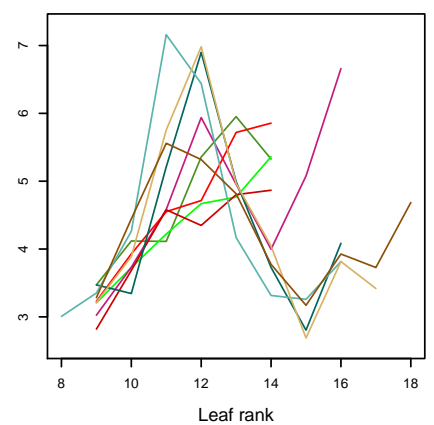

2016

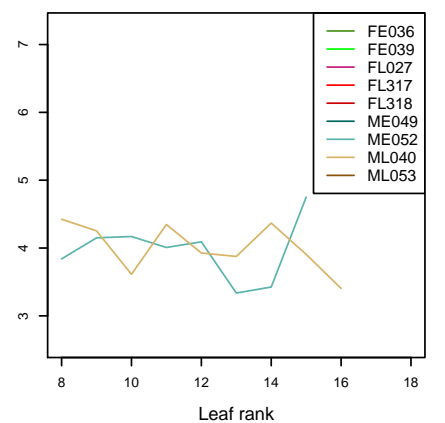

Figure 4: Estimates of instant phyllochron. Each plot corresponds to a year; Colors correspond to the different genotypes; Lines join estimates $\left(\widehat{\mu}_{y, l s g, f}\right)_{f=f_{\min }^{y, l s g}+1, \ldots, f_{\max }^{y, l s g}}$ from the same genotype $l s g$ at successive leaf ranks. 

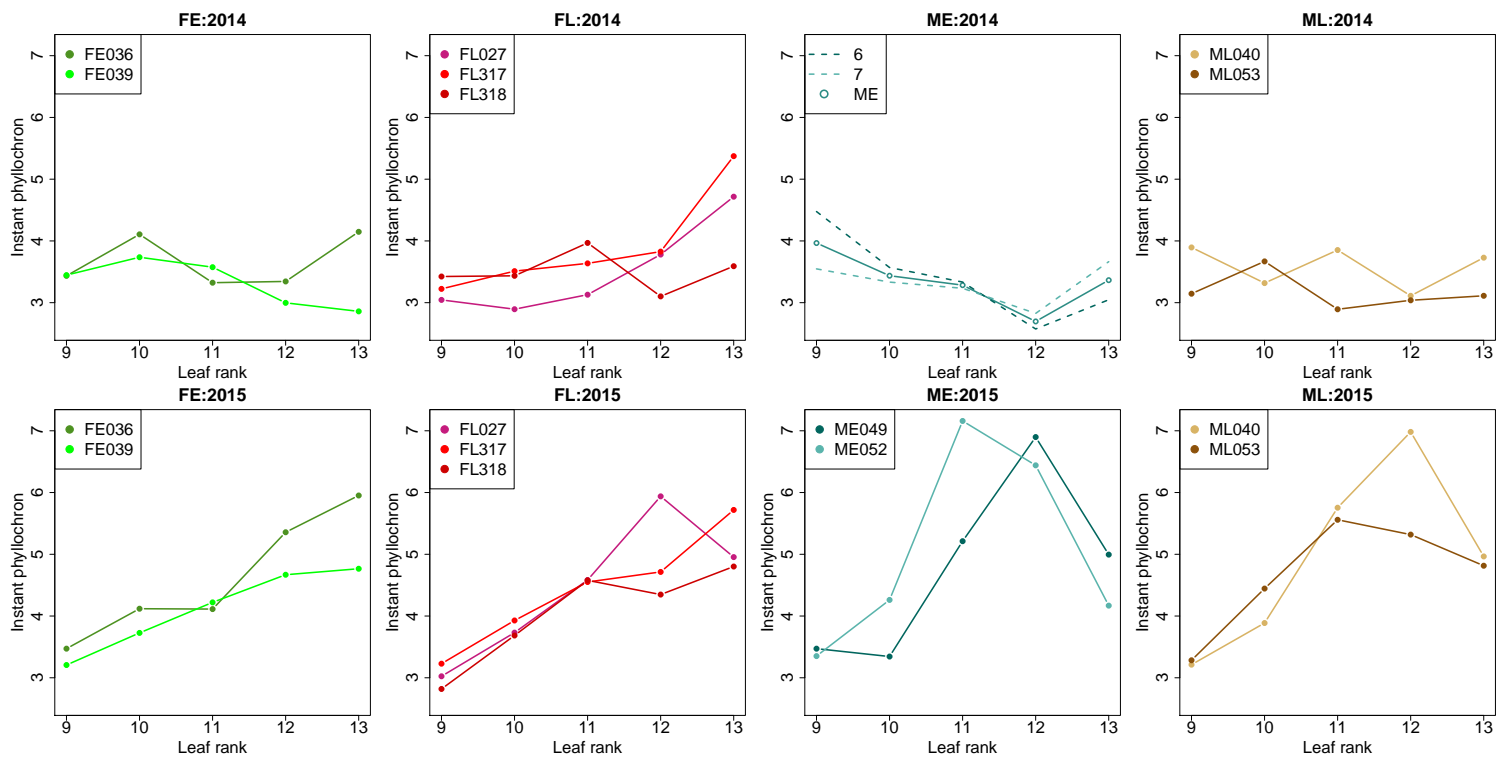

Figure 5: Genotypic and selection effects on instant phyllochron. Instant phyllochrons of all genotypes within selection groups. Columns correspond to selection populations and row to years. When differences between genotypes within selection population were not significant, the phyllochron estimates on the whole selection groups was represented. 

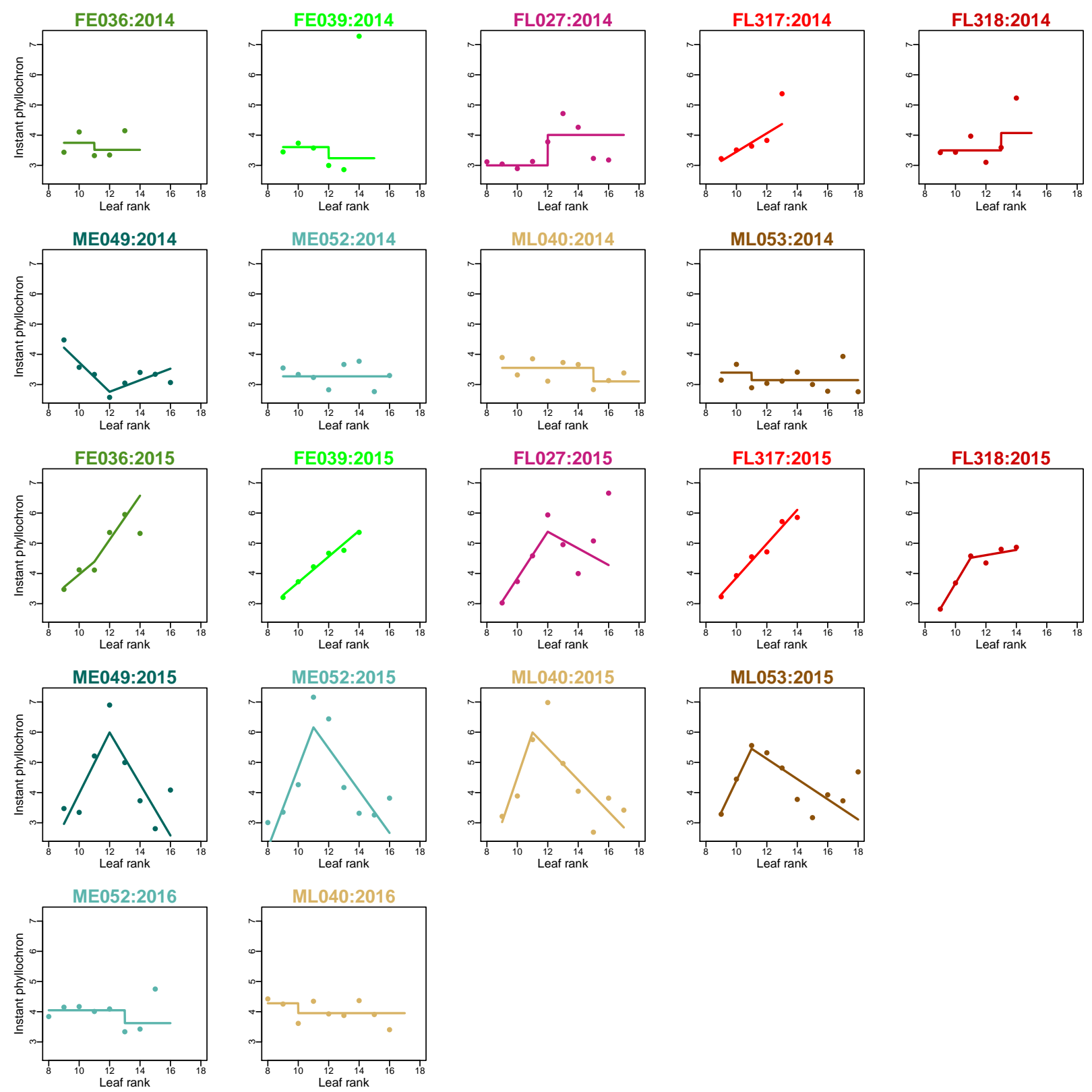

Figure 6: Best parametric sub-models. Each plot shows the instant phyllochron $\left(\widehat{\mu}_{y, l s g, f}\right)_{f=f_{\min }^{y, l s g}+1, \ldots, f_{\max }^{y, l s g}}$ in degree days for each genotype and each year. Dots correspond to the estimates under the complete model, while straight line display the estimate under the best parametric sub-model. 


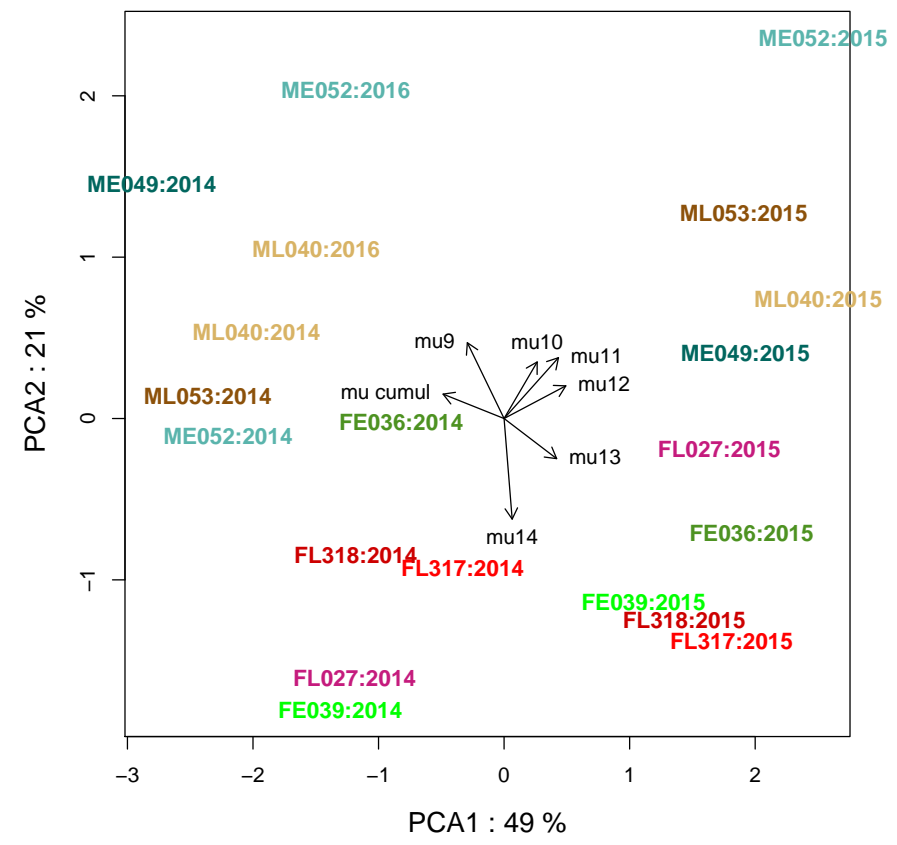

Figure 7: Principal Component Analysis of seasonal variations of the phyllochron. Variables are the average time $\widehat{\bar{\mu}}_{y, l s g, 8}$ between sowing and appearance of leaf 8 and the instant phyllochrons $\left(\widehat{\mu}_{y, l s g, 9}, \ldots, \widehat{\mu}_{y, l s g, 14}\right)$ between leaf ranks nine and 14 . Individuals are the genotype-year combinations. Individuals are represented on the first two axes of PCA and the colors correspond to the genotypes. Correlations between the variables and the PCA axes are represented by the black arrows using an arbitrary scale.

2014

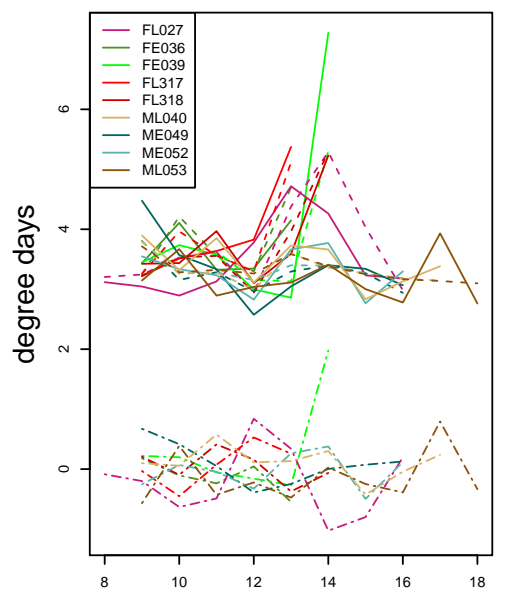

2015

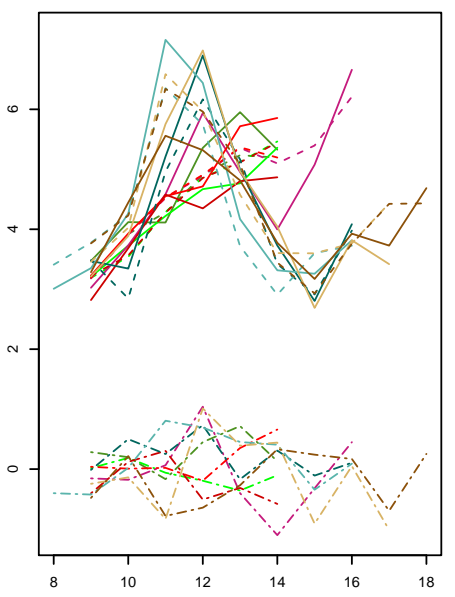

2016

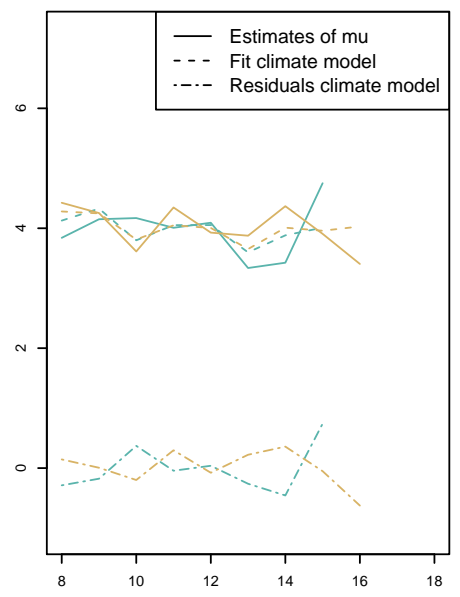

Figure 8: Fitted values and residuals of the climate model with selected variables and windows. Each plot corresponds to a year and each color to a genotype. Fitted values with the climate model $m_{1}^{\text {best }}$ (solid line), original estimates of $\mu$ (dashed line) and residuals of model $m_{1}^{\text {best }}$ (dot-dashed line) are displayed as a function of the leaf rank. 

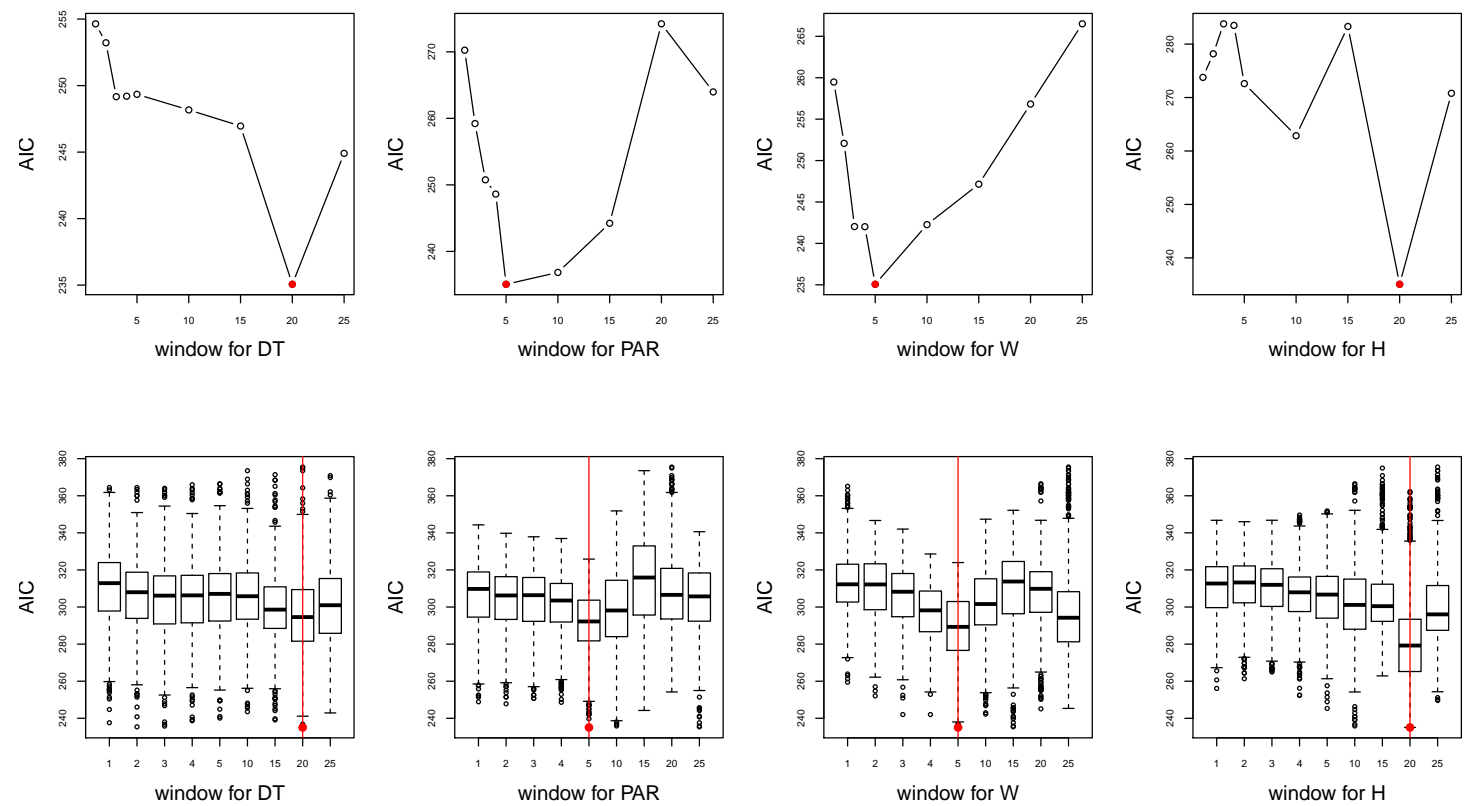

Figure 9: AIC of the climatic model as window time varies. For each selected variables, (DT = Dewpoint Temperature, PAR = Photosynthetically Active Radiation, $\mathrm{W}=\mathrm{Wind}, \mathrm{H}=$ Humidity), the AIC is displayed when the window time of the variable varies. First row: windows for the other variables are fixed to the optimal value; second row: all combinations of windows for the other variables are considered. Red bullet and line represent the optimal value. 


\section{Supplementary materials}

\section{A Monte Carlo Expectation Maximisation Algorithm}

Consider a given genotype $l s g$ (or higher level genotypic group) and year $y$, the indexes $l s g$ and $y$ are omitted in this section. For a plant $p$, let $\left(t_{p, 1}, \ldots, t_{p, N_{p}}\right)$ be the monitoring times (with time origin at sowing) and $X_{p, j}$ the number of leaves of plant $p$ at time $t_{p, j}$. For each plant and leaf rank, the observations indicate that leaf $f$ appeared at some time between the last observation date when the plant had at most $(f-1)$ leaves, and the first observation date when the plants had at least $f$ leaves. We denote by $\nu_{p, f}$ and $\tau_{p, f}$ respectively these two dates:

$$
\begin{aligned}
& \nu_{p, f}= \begin{cases}\max \left\{t_{p, j}, j \text { s.t. } X_{p, j} \leq f\right\} & \text { if } \min \left(X_{p, j}\right) \leq f \\
0 & \text { otherwise }\end{cases} \\
& \tau_{p, f}= \begin{cases}\min \left\{t_{p, j}, j \text { s.t. } X_{p, j}>f\right\} & \text { if } \max \left(X_{p, j}\right)>f \\
+\infty & \text { otherwise }\end{cases}
\end{aligned}
$$

The likelihood of the observations involves an integral of dimension $f_{\max }-f_{\min }+1$, thus a direct maximisation would be intractable. Thus, we considered a Monte-Carlo Expectation Maximisation algorithm, wihere the complete data (unobserved) are $\mathbf{H}_{p}=\left(H_{p, f}\right)_{p=1, \ldots, n, f=f_{\min }, \ldots, f_{\max }}$

- Initialisation. Proxies of the $\left\{H_{p, f}\right\}_{p, f}$ are computed as:

$$
\widehat{H_{p, f}}=\frac{1}{2}\left(\nu_{p, f}+\tau_{p, f}\right), \quad f=f_{\min }, \ldots, f_{\max }
$$

for all $(p, f)$ such that $\nu_{p, f}>0$ and $\tau_{p, f}<\infty$, and the initial values of $\mu_{f}$ and $\sigma_{f}$ are inferred as robust estimators of mean and variance based on the proxies.

- Monte-Carlo E-step. Let $\Theta^{(m)}=\left(\mu^{C,(m)}, \sigma^{C,(m)}, \mu_{f}^{(m)}, \sigma_{f}^{(m)}\right)_{f=f_{\min }+1, \ldots, f_{\max }}$ be the current value of the parameters. For each $p$ a sample $\left(\mathbf{H}_{p, r}^{(m)}\right)_{r=1, \ldots, N}$ is generated from the conditional distribution:

$$
\mathbb{P}_{\Theta(m)}\left[\mathbf{H}_{p} \mid H_{p, f} \in\left[\nu_{p, f}+\tau_{p, f}\right), f=f_{\min }, \ldots, f_{\max }\right]
$$

namely a truncated multivariate Gaussian distribution. Computation time of classic rejection methods dramatically increase with the dimension, nevertheless specific are available for the truncated multivariate distribution. We used the $\mathrm{R}$ package TruncatedNormal (Botev and Belzile, 2020) well adapted when the truncated region is in the tail of the distribution.

- M-step. For $f=f_{\min }+1, \ldots, f_{\max }$, let $Y_{p, r, f}^{(m)}=H_{p, r, f}^{(m)}-Y_{p, r, f-1}^{(m)}$. The new value of the parameters $\Theta^{(m+1)}$ maximises

$$
\sum_{f=f_{\min }+1}^{f_{\max }}\left(\sum_{p=1}^{n} \sum_{r=1}^{N} \log \phi_{\mu_{f}, \sigma_{f}}\left(Y_{p, r, f}^{(m)}\right)\right)+\sum_{p=1}^{n} \sum_{r=1}^{N} \log \phi_{\mu_{f_{\min }}^{C}, \sigma_{f_{\min }}^{C}}\left(H_{p, r, f_{\min }}^{(m)}\right)
$$

with $\phi_{\mu, \sigma}$ the density of the normal distribution with mean $\mu$ and standard deviation $\sigma$. This problem is equivalent to a simple maximum likelihood estimation of a multivariate Gaussian distribution with diagonal covariance matrix, thus:

$$
\begin{aligned}
\mu_{f}^{(m+1)} & =\frac{1}{n N} \sum_{p=1}^{n} \sum_{r=1}^{N} Y_{p, r, f}^{(m)}, \quad f=f_{\min }+1, \ldots, f_{\max } \\
\mu^{C,(m+1)} & =\frac{1}{n N} \sum_{p=1}^{n} \sum_{r=1}^{N} H_{p, r, f_{\min }}^{(m)} \\
\left(\sigma_{f}^{(m+1)}\right)^{2} & =\frac{1}{n N} \sum_{p=1}^{n} \sum_{r=1}^{N}\left(Y_{p, r, f}^{(m)}\right)^{2}-\left(\mu_{f}^{(m+1)}\right)^{2}, \quad f=f_{\min }+1, \ldots, f_{\max } \\
\left(\sigma^{C,(m+1)}\right)^{2} & =\frac{1}{n N} \sum_{p=1}^{n} \sum_{r=1}^{N}\left(H_{p, r, f_{\min }}^{(m)}\right)^{2}-\left(\mu^{C,(m+1)}\right)^{2}
\end{aligned}
$$


The algorithm is iterated until stabilisation of the parameters.

\section{B Parametric sub-models}

For each genotype $l s g$ and year $y$, we consider the following parametric models for the average interval times between leaves $\mu_{y, l s g, f_{\min }^{l s g}+1}, \ldots, \mu_{y, l s g, f_{\max }^{y, l s g}}$ as a function of leaf rank:

- Constant: $\mu_{y, l s g, f}=a_{y, l s g}, \forall f=f_{\min }^{y, l s g}+1, \ldots, f_{\max }^{y, l s g} \quad$ (mod-ct)

- Linear: $\mu_{y, l s g, f}=a_{y, l s g} \times f+b_{y, l s g}, \forall f=f_{\min }^{y, l s g}+1, \ldots, f_{\max }^{y, l s g} \quad$ (mod-lin)

- Piecewise constant: $\mu_{y, l s g, f}=a_{l s g}+b_{y, l s g} \amalg_{f>\kappa}, \forall f=f_{\min }^{y, l s g}+1, \ldots, f_{\max }^{y, l s g}$, for $\kappa=f_{\min }+$ $2, \ldots, f_{\max }-2 \quad(\bmod -\operatorname{pct}(\kappa))$

- Piecewise linear: $\mu_{y n l s g, f}=a_{y, l s g}+b_{y, l s g}\left(f \mathbb{1}_{f \leq \kappa}+\kappa \mathbb{I}_{f>\kappa}\right)+c_{y, l s g}(f-\kappa) \mathbb{I}_{f>\kappa}, \forall f=f_{\min }^{y, l s g}+$ $1, \ldots, f_{\max }^{y n l s g}$, for $\kappa=f_{\min }+3, \ldots, f_{\max }-3(\bmod -\operatorname{plin}(\kappa))$

In order to test the classic hypothesis of constant leaf appearance rate which corresponds to (mod-ct), for each genotype we compared models (mod-lin), (mod-pct $(\kappa)))$, (mod-plin $(\kappa))$ with (mod-ct). First, (mod-ct) was compared to all parametric models and to the complete model using the $\chi^{2}$-likelihood ratio test; if all $p>0.01$, (mod-ct) is selected; otherwise, the parametric model with the largest AIC is selected.

Monte Carlo EM algorithm for the parametric sub-models. We omit the indexes $y$ and $l s g$. The parametric models we considered are equivalent to imposing a linear constraint:

$$
B_{q}\left(\mu_{f_{\min }+1}, \ldots, \mu_{f_{\max }}\right)=0
$$

with $B_{q}$ a $q \times s$-matrix, $s=f_{\max }-f_{\min }$ and $q<s$. The parameter $\mu^{C}$ is unconstrained.

- Constant model (mod-ct):

$$
B_{q}=\left(\begin{array}{ccccc}
1 & -1 & 0 & \cdot & 0 \\
1 & 0 & -1 & \cdot & 0 \\
& \cdot & \cdot & \cdot & \\
1 & 0 & \cdot & 0 & -1
\end{array}\right)
$$

- Linear model: $B_{q}$ is the $(s-2) \times s$ matrix such that for every $j>2$

$$
\left\{\begin{array}{l}
B_{q}[j-2,1]=j-2 \\
B_{q}[j-2,2]=1-j \\
B_{q}[j-2, j]=1
\end{array}\right.
$$

and the other coefficients are zero.

- Piecewise constant $(\bmod -\operatorname{pct}(\kappa))$ : let $\kappa^{\prime}=\kappa-f_{\min }$, then $B_{q}$ is the $(s-2) \times s$ matrix with,

$$
\begin{gathered}
B_{q}[j-1,1]=1, \quad B_{q}[j-1, j]=-1 \quad \text { if } \quad j=2, \ldots, \kappa^{\prime} \\
B_{q}[j-1, s]=1, \quad B_{q}[j-1, j]=-1 \quad \text { if } \quad j=\kappa^{\prime}, \ldots, s-1
\end{gathered}
$$

and the other coefficients are zero.

- Piecewise linear: let $\kappa^{\prime}=\kappa-f_{\min }$ $B_{q}$ is the $(s-3) \times s$ matrix such that for every $2<j \leq \kappa^{\prime}$

$$
\left\{\begin{array}{l}
B_{q}[j-2,1]=j-2 \\
B_{q}[j-2,2]=1-j \\
B_{q}[j-2, j]=1
\end{array}\right.
$$




\begin{tabular}{lll}
\hline Model & $\mu^{C}$ parameters & $\mu$ parameters \\
\hline$M_{00}^{\sigma}$ & $\forall(l, s, g), \quad\left(\mu_{l s g}^{C}, \sigma_{l s g}^{C}\right)=\left(\mu^{C}, \sigma_{l}^{C}\right)$ & $\forall(l, s, g), \quad\left(\mu_{l s g}, \sigma_{l s g}\right)=\left(\mu, \sigma_{l}\right)$ \\
$M_{11}^{\sigma}$ & $\forall(s, g), \quad\left(\mu_{l s g}^{C}, \sigma_{l s g}^{C}\right)=\left(\mu_{l}^{C}, \sigma_{l s}^{C}\right)$ & $\forall(s, g), \quad\left(\mu_{l s g}, \sigma_{l s g}\right)=\left(\mu_{l}, \sigma_{l s}\right)$ \\
$M_{22}^{\sigma}$ & $\forall(g),\left(\mu_{l s g}^{C}, \sigma_{l s g}^{C}\right)=\left(\mu_{l s}^{C}, \sigma_{l s g}^{C}\right)$ & $\left(\mu_{l s g}, \sigma_{l s}\right)=\left(\mu_{l s g}, \sigma_{l s g}\right)$ \\
\hline
\end{tabular}

Table S1: Models in which the mean parameters depend on grouping level $j-1$ and the variance parameters on grouping level $j$

and for every $\kappa^{\prime}+1<j \leq s$

$$
\left\{\begin{array}{l}
B_{q}[j-2,1]=\kappa-2 \\
B_{q}[j-2,2]=1-\kappa \\
B_{q}[j-2, j]=1 \\
B_{q}[j-2, \kappa]=j-\kappa \\
B_{q}[j-2, \kappa+1]=\kappa-j
\end{array}\right.
$$

and the other coefficients are zero.

The Monte-Carlo E-step is identical to the case of unconstrained $\mu$. The M-step corresponds to the maximum likelihood estimation problem from a multivariate Gaussian distributed sample under linear constraints on the mean, which admits an explicit expression as follows (Zoppé et al., 2001). Let $B_{s-q}$ be any matrix such that

$$
B=\left(\begin{array}{c}
B_{s-q} \\
B_{q}
\end{array}\right)
$$

is invertible. Then, let

$$
S^{(m)}=\frac{1}{n N} \sum_{p=1}^{n} \sum_{r=1}^{N} B\left(\tilde{\mathbf{Y}}_{p, r}^{(m)}-\overline{\tilde{\mathbf{Y}}}^{(m)}\right)\left(\tilde{\mathbf{Y}}_{p, r}^{(m)}-\overline{\tilde{\mathbf{Y}}}^{(m)}\right)^{t} B^{t}=\left(\begin{array}{cc}
S_{s-q, s-q} & S_{s-q, q} \\
S_{q, s-q} & S_{q, q}
\end{array}\right)
$$

where $\tilde{\mathbf{Y}}_{p, r}^{(m)}=\left(Y_{p, r, f}^{(m)}\right)_{f=f_{\min }+1, \ldots, f_{\max }}, \overline{\tilde{\mathbf{Y}}}=(1 / n N) \sum_{p=1}^{n} \sum_{r=1}^{N} \tilde{\mathbf{Y}}_{p, r}$, and $S_{a, b}$ are submatrices of $S$ with dimension $a \times b$. Let $\overline{\tilde{\mathbf{Y}}}_{s-q}\left(\right.$ resp. $\left.\overline{\tilde{\mathbf{Y}}}_{q}\right)$ be the subvector of $\overline{\tilde{\mathbf{Y}}}$ with the $s-q$ first (resp. the $q$ last) coordinates of $\overline{\tilde{\mathbf{Y}}}$, then

$$
\left(\mu_{f_{\min }+1}^{(m+1)}, \ldots, \mu_{f_{\max }}^{(m+1)}\right)=B^{-1}\left(\begin{array}{c}
\overline{\tilde{\mathbf{Y}}}_{s-q}-S_{q, q}^{-1} S_{s-q, q} \overline{\mathbf{Y}}_{q} \\
0
\end{array}\right)
$$

Finally, $\left(\mu^{C,(m+1)}, \sigma^{C,(m+1)}, \sigma^{(m+1)}\right)$ are given by equation (4).

\section{Are genotypic group effect due to differences in the average phyl- lochron?}

The differences of phyllochron between genotypic groups (genotypes, selection populations and inbred lines) could originate from differences in the average phyllochron $\left(\mu^{C}, \mu\right)$ and/or on the standard deviations $\left(\sigma^{C}, \sigma\right)$. In this subsection, we perform additional comparisons to ensure that the genotypic effect indeed impacts the average phyllochron. In addition to models $M_{j-1, j-1}$ and $M_{j, j}$ in Table 1 , we considered models $M_{j-1, j-1}^{\sigma}$ in which the mean parameters depend on grouping level $j-1$ and the variance parameters on grouping level $j$ (Table S1)

The comparison of models $M_{j, j}$ and $M_{j-1, j-1}^{\sigma}$ enables to test the effect of genotypic grouping on the mean parameters $\left(\mu_{y, l s g, f}\right)$ only, regardless of its effect on variance parameters $\left(\sigma_{y, l s g, f}\right)$. Results in Table S2 indicate that these comparisons lead to similar p-values than the comparison $M_{j, j} / M_{j-1, j-1}$. Moreover, the estimates of $\left(\mu_{y, l s g, f}\right)$ computed under models $M_{j-1, j-1}$ and $M_{j-1, j-1}^{\sigma}$ are very similar (Figure S2). Therefore, the observed genotypic groups effects are due, in a significant part, to differences on the average phyllochron $\left(\mu_{y, l s g}^{C}, \mu_{y, l s g, f}\right)$ rather than on the nuisance parameters $\left(\sigma_{y, l s g}^{C}, \sigma_{y, l s g, f}\right)$. 


\section{Criteria for model comparison - Evaluation through permutation test.}

Intially, we considered three criteria for model comparisons.

- the AIC criterion $-2 \ell+2 p$ with $\ell$ the log-likelihood of the observations and $p$ the number of parameters in the model. The smaller the better.

- the BIC criterion $-2 \ell+p \times \log n$ with $n$ the sample size (number of plants). The smaller the better. As soon as the sample size is larger than $8, \mathrm{BIC}$ is more conservative than AIC.

- The p-value of the $\chi^{2}$-likelihood ratio test.

These three criteria rely on asymptotic heuristics, and there is no obvious choice a priori. On the contrary, permutation tests are unbiased but too time consuming to be used for all comparisons. Thus, in order to evaluate the relevance of the three criteria for finite sample size, we used a permutation test with $N=200$ permutations for the comparison $M_{22} / M_{33}$ for the two genotypes of the group F252-Early. On the non-permuted data, AIC selected $H_{1}$ (1904 versus 1915 ) indicating a genotypic effect, while BIC (which is more conservative by definition) selected $H_{0}$ (1954 vs 1982), indicating no genotypic effect. The $\chi^{2}$-likelihood ratio test had a significant p-value $\left(5.10^{-5}\right)$, leading to the same conclusion that the AIC criterion. Therefore, we wondered if BIC criterion is too conservative, or if $\mathrm{AIC}$ and $\chi^{2}$-test over-estimated the genotypic effect.

On the one hand, the unbiased permutation test had a p-value of 0.02 , coherent with the $\chi^{2}-\log$ likelihood test, even less significant. Besides, the quantile 0.05 of the empirical distribution of the $\chi^{2}$ p-value on the resampled data, equal to 0.013 , could be considered as a conservative heuristic threshold, since tests involving more plants (e.g. selection or line effect) are expected to be less biased. Nevertheless, given the very weak p-values obtained on the data, this distinction would not dramatically change the conclusions. On the other hand, the AIC criterion proved to be reliable since it mostly selects $M_{22}$ on the resampled data ( $94.5 \%$ of the permutations), even slightly too selective; similarly to $\chi^{2}$-likelihood test. Finally, the BIC criterion appeared too conservative, as it did not allowed to detect a significant genotypic effect with the original genotypes.

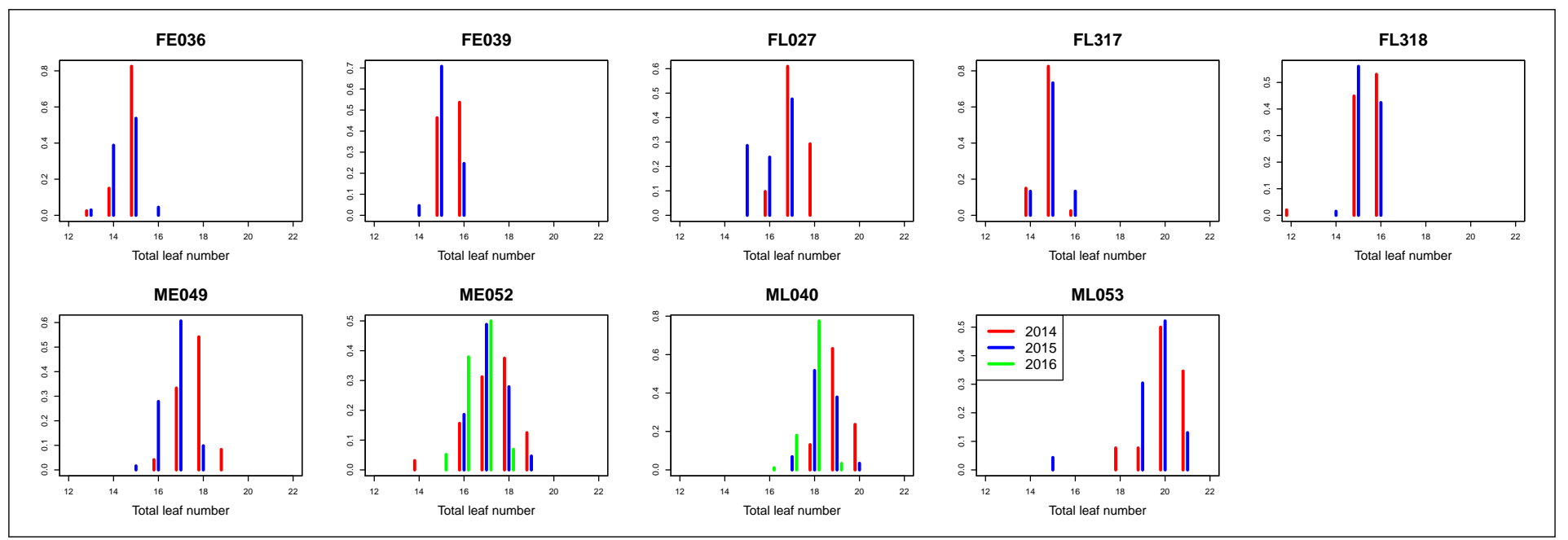

Figure S1: Distribution of the total leaf number for all plants by genotype and by year. 
All plants:2014

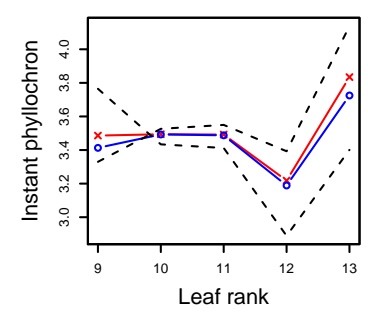

F252-Early:2014

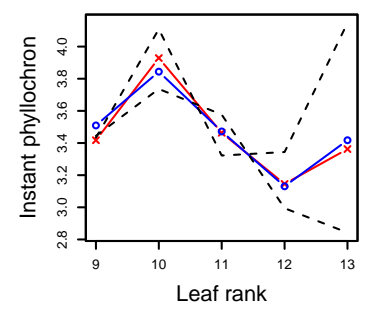

All plants:2015

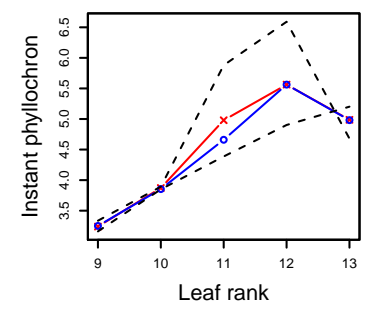

F252-Early:2015

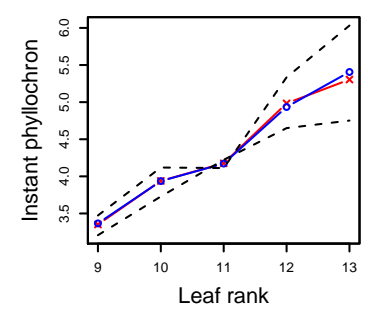

F252:2014

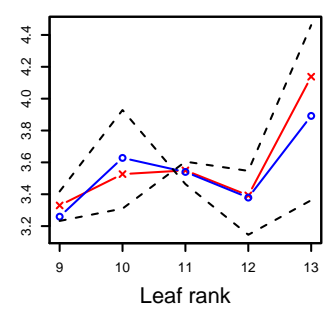

F252-Late:2014

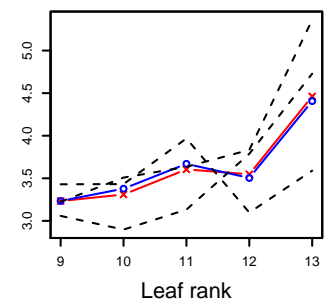

F252:2015

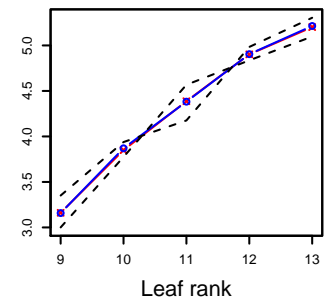

F252-Late:2015

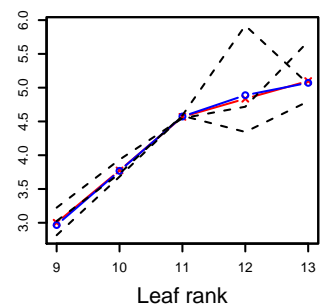

MBS:2014

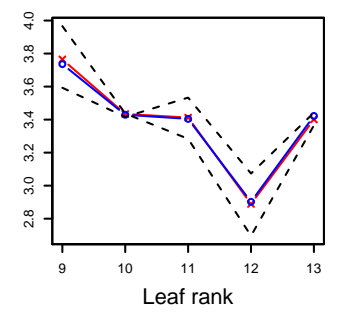

MBS-Early:2014

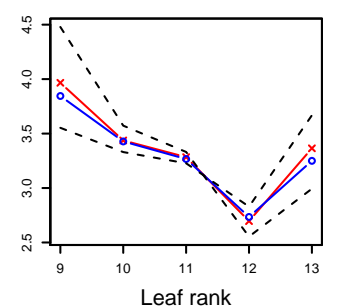

MBS:2015

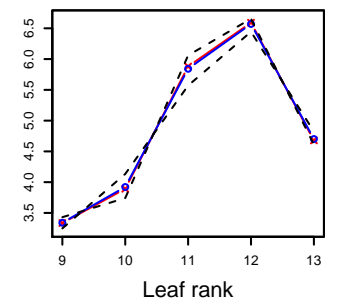

MBS-Early:2015

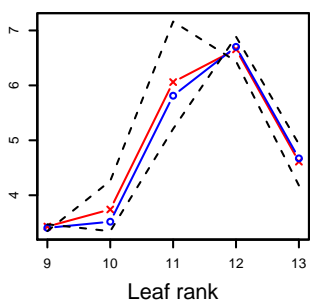

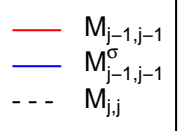

MBS-Late:2014

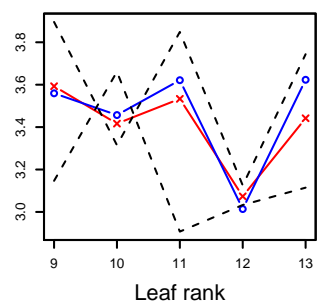

MBS-Late:2015

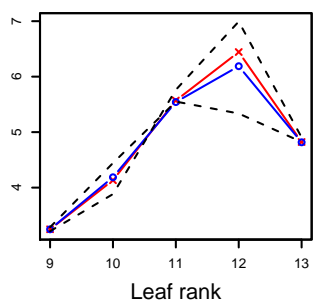

Figure S2: Estimates of instant phyllochron for models $M_{j-1, j-1}\left(\left(\mu^{C}, \mu, \sigma^{C}, \sigma\right)\right.$ depend on the grouping level $j-1$, red), $M_{j, j}\left(\left(\mu^{C}, \mu, \sigma^{C}, \sigma\right)\right.$ depend on the grouping level $j$, dashed lines $)$ and $M_{j-1, j-1}^{\sigma}\left(\left(\mu^{C}, \mu\right)\right.$ depend on the grouping level $j-1$ and $\left(\sigma^{C}, \sigma\right)$ depend on the grouping level $j$, blue). Models are described in Tables 1 and S1. Rows 1 and 2 correspond to year 2014, and rows 3 and 4 to 2015. For each year, plots entitled All plants correspond to $j=1$, plots F252 and MBS to $j=2$, and plots F252-Early, F252-Late, MBS-Early and MBS-Late to $j=3$.

2014

\begin{tabular}{|rrr|}
\hline & $M_{j, j} / M_{j-1, j-1}$ & $M_{j, j} / M_{j-1, j-1}^{\sigma}$ \\
\hline all & $<\mathrm{e}-16$ & $7.3 \mathrm{e}-15$ \\
$\mathrm{~F}$ & $2.00 \mathrm{e}-12$ & $1.8 \mathrm{e}-09$ \\
$\mathrm{M}$ & $4.20 \mathrm{e}-02$ & $7.2 \mathrm{e}-02$ \\
Fearly & $6.60 \mathrm{e}-05$ & $3.6 \mathrm{e}-05$ \\
Flate & $5.70 \mathrm{e}-12$ & $1.1 \mathrm{e}-9$ \\
Mearly & $6.00 \mathrm{e}-02$ & $7.3 \mathrm{e}-02$ \\
Mlate & $3.80 \mathrm{e}-05$ & $6.3 \mathrm{e}-06$ \\
\hline
\end{tabular}

2015

\begin{tabular}{|rrr|}
\hline & $M_{j, j} / M_{j-1, j-1}$ & $M_{j, j} / M_{j-1, j-1}^{\sigma}$ \\
\hline all & $<\mathrm{e}-16$ & $<\mathrm{e}-16$ \\
$\mathrm{~F}$ & $3.0 \mathrm{e}-03$ & $1.7 \mathrm{e}-02$ \\
$\mathrm{M}$ & $1.3 \mathrm{e}-05$ & $2.0 \mathrm{e}-04$ \\
Fearly & $4.9 \mathrm{e}-04$ & $6.8 \mathrm{e}-05$ \\
Flate & $3.7 \mathrm{e}-09$ & $9.4 \mathrm{e}-06$ \\
Mearly & $<\mathrm{e}-16$ & $<\mathrm{e}-16$ \\
Mlate & $1.5 \mathrm{e}-04$ & $2.0 \mathrm{e}-05$ \\
\hline
\end{tabular}

Table S2: $\quad \chi^{2}$ likelihood ratio p-value for genotypic group effect on the mean and variance of the phyllochron $\left(M_{j, j} / M_{j-1, j-1}\right)$ and on the average phyllochron $\mu$ only $\left(M_{j, j} / M_{j-1, j-1}^{\sigma}\right)$. 


\section{Dendrogram of climatic variables}

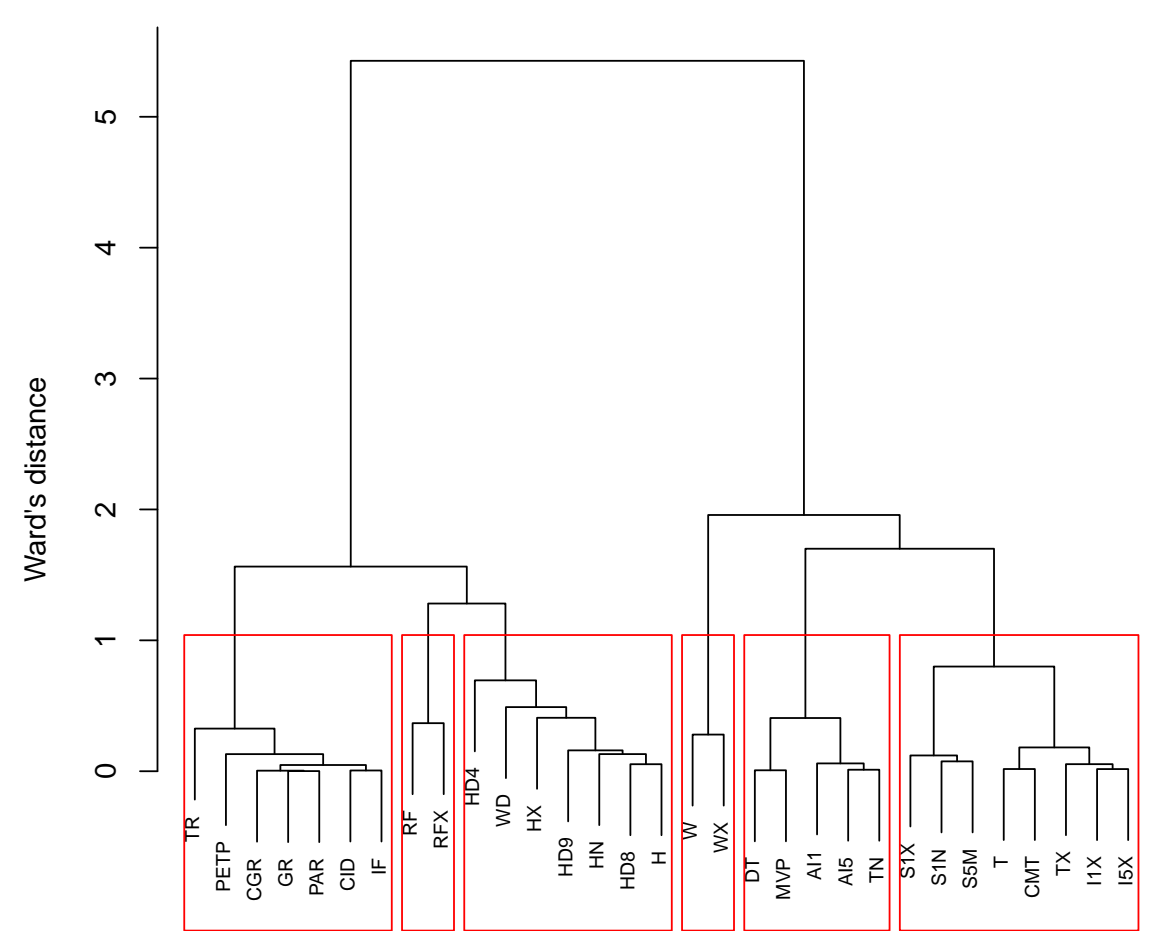

Climatic variables

Figure S3: Dendrogram and clusters identified among climatic variables. $\mathrm{TR}=$ temperature range, $\mathrm{PETP}=$ Penman evapotranspiration, $\mathrm{CGR}=$ calculated global radiation, $\mathrm{GR}=$ global radiation, $\mathrm{PAR}=$ photosynthetically active radiation, $\mathrm{CID}=$ calculated sunshine duration, $\mathrm{IF}=$ percentage of sunshine, $\mathrm{RF}=$ rain falls, $\mathrm{RFX}=$ maximum rain falls, $\mathrm{HD} 4=>40 \%$ humidity duration, $\mathrm{WD}=$ wetness duration, $\mathrm{HX}=$ maximal humidity, HD9 $=>90 \%$ humidity duration, HN $=$ minimum humidity,HD8 $=>80 \%$ humidity duration, $\mathrm{H}=$ atmospheric humidity, $\mathrm{W}=$ wind speed, WX $=$ maximum wind speed, DT $=$ dewpoint temperature, $\mathrm{MVP}=$ mean vapor pressure, $\mathrm{AI} 1=$ actinothermic index $10 \mathrm{~cm}, \mathrm{AI} 5=$ actinothermic index $50 \mathrm{~cm}, \mathrm{TN}=$ minimum temperature, $\mathrm{S} 1 \mathrm{X}=$ soil maximum temperature $50 \mathrm{~cm}, \mathrm{~S} 1 \mathrm{~N}=$ soil minium temperature $10 \mathrm{~cm}, \mathrm{~S} 5 \mathrm{M}=$ soil temperature $50 \mathrm{~cm}, \mathrm{~T}=$ mean temperature, $\mathrm{CMT}=$ calculated mean temperature, $\mathrm{TX}=$ maximum temperature, $\mathrm{I} 1 \mathrm{X}=$ maximum actinothermic index $10 \mathrm{~cm}, \mathrm{I} 5 \mathrm{X}=$ maximum actinothermic index $50 \mathrm{~cm}$. 


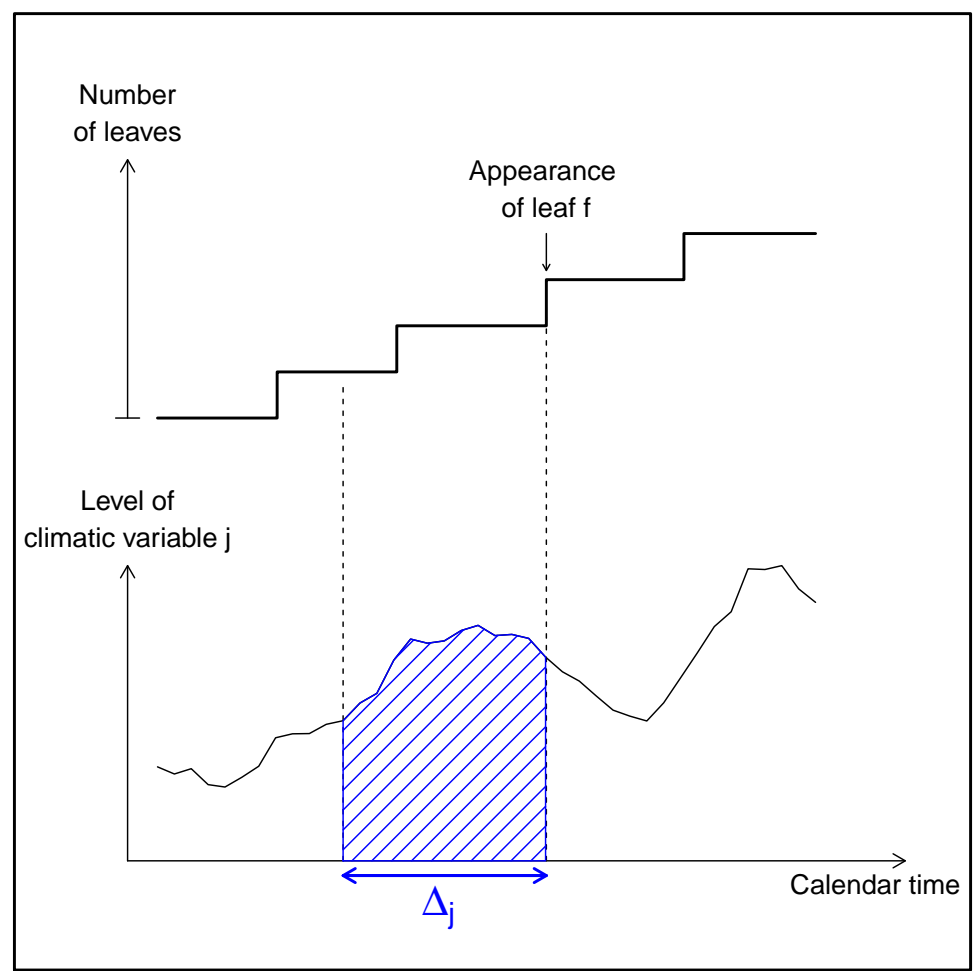

Figure S4: Time window in the climatic variable model $m_{1}$. The upper graph displays the average phyllochron for genotype $l s g$ on year $y$, and the lower graph a climatic variable. The average value $C_{y, l s g, f, j}^{\left(\Delta_{j}\right)}$ of the climatic variable on an interval $\Delta_{j}$ before appearance of leaf $f$ corresponds to the hatched area normalised by $\Delta_{j}$. 